UWThPh-2017-11

\title{
Renormalization and radiative corrections to masses in a general Yukawa model
}

\author{
M. Fox*, W. Grimus ${ }^{\ddagger}$ and M. Löschner ${ }^{\S}$ \\ University of Vienna, Faculty of Physics \\ Boltzmanngasse 5, A-1090 Vienna, Austria
}

October 2, 2017

\begin{abstract}
We consider a model with arbitrary numbers of Majorana fermion fields and real scalar fields $\varphi_{a}$, general Yukawa couplings and a $\mathbb{Z}_{4}$ symmetry that forbids linear and trilinear terms in the scalar potential. Moreover, fermions become massive only after spontaneous symmetry breaking of the $\mathbb{Z}_{4}$ symmetry by vacuum expectation values (VEVs) of the $\varphi_{a}$. Introducing the shifted fields $h_{a}$ whose VEVs vanish, $\overline{\mathrm{MS}}$ renormalization of the parameters of the unbroken theory suffices to make the theory finite. However, in this way, beyond tree level it is necessary to perform finite shifts of the tree-level VEVs, induced by the finite parts of the tadpole diagrams, in order to ensure vanishing one-point functions of the $h_{a}$. Moreover, adapting the renormalization scheme to a situation with many scalars and VEVs, we consider the physical fermion and scalar masses as derived quantities, i.e. as functions of the coupling constants and VEVs. Consequently, the masses have to be computed order by order in a perturbative expansion. In this scheme we compute the selfenergies of fermions and bosons and show how to obtain the respective one-loop contributions to the tree-level masses. Furthermore, we discuss the modification of our results in the case of Dirac fermions and investigate, by way of an example, the effects of a flavour symmetry group.
\end{abstract}

\footnotetext{
*E-mail: a0905865@unet.univie.ac.at

${ }^{\ddagger}$ E-mail: walter.grimus@univie.ac.at

${ }^{\S}$ E-mail: maximilian.loeschner@univie.ac.at
} 


\section{Introduction}

Thanks to the results of the neutrino oscillation experiments - see for instance [1, 2] it is now firmly established that at least two light neutrinos have a nonzero mass and that there is a non-trivial lepton mixing matrix or PMNS matrix in analogy to the quark mixing matrix or CKM matrix. The surprisingly large mixing angles in the PMNS matrix have given a boost to model building with spontaneously broken flavour symmetriesfor recent reviews see [3]. Many interesting results have been discovered, however, no favoured scenario has emerged yet. Moreover, predictions of neutrino mass and mixing models refer frequently to tree-level computations. It would thus be desirable to check the stability of such predictions under radiative corrections. In the case of renormalizable models one has a clear-cut and consistent method to remove ultraviolet (UV) divergences and to compute such corrections.

However, there is the complication that the envisaged models always have a host of scalars and often complicated spontaneous symmetry breaking (SSB) of the flavour group. This makes it impossible to replace all Yukawa couplings by ratios of masses over vacuum expectation values (VEVs), as done for instance in the renormalization of the Standard Model. Of course, one could replace part of the Yukawa coupling constants by masses, but this would make the renormalization procedure highly asymmetric. In this paper we suggest to make such models finite by $\overline{\mathrm{MS}}$ renormalization of the parameters of the unbroken model and to perform finite VEV shifts at the loop level in order to guarantee vanishing scalar one-point functions of the shifted scalar fields [4]. Additionally, we introduce finite field strength renormalization for obtaining on-shell selfenergies. In this way, all fermion and scalar masses are derived quantities and functions of the parameters of the model.

In the usual approach to renormalization of theories with SSB and mixing [5] one has counterterms for masses, quark and lepton mixing matrices - see for instance [6, 7, and tadpoles - see for instance [8, 9] 1 . We stress that in our approach there are no such counterterms because we use an alternative approach tailored to the situation with a proliferation of scalars and VEVs.

In order to present the renormalization scheme in a clear and compact way, we consider a toy model which has

- an arbitrary number of Majorana or Dirac fermions,

- an arbitrary number of neutral scalars,

- a $\mathbb{Z}_{4}\left(\mathbb{Z}_{2}\right)$ symmetry which forbids Majorana (Dirac) fermion masses before SSB2 and

- general Yukawa interactions.

\footnotetext{
${ }^{1}$ There are other treatments of tadpoles adapted to the theory where they occur, see for instance reference [10] for the MSSM and [11, 12] where the issue of gauge invariance is discussed.

${ }^{2}$ This is motivated by the Standard Model where - before SSB - fermion masses as well as linear and trilinear terms in the scalar potential are absent due to the gauge symmetry.
} 
We put particular emphasis on the treatment of tadpoles. Since radiative corrections in this model are already finite due to $\overline{\mathrm{MS}}$ renormalization with the counterterms of the unbroken theory, also the sum of all tadpole contributions, i.e. the loop contributions and those induced by the counterterms of the unbroken theory, is finite. However, tadpoles introduce finite VEV shifts which have to be taken into account for instance in the selfenergies. Eventually, the finite VEV shifts also contribute to the radiative corrections of the tree-level masses 3 We also focus on Majorana fermions, having in mind that neutrinos automatically obtain Majorana nature through the seesaw mechanism [13].

An attempt at a renormalization scheme - with one fermion and one scalar fieldalong the lines discussed here has already been made in [14]; however, the treatment of the VEV in this paper cannot be generalized to the case of more than one scalar field.

The paper is organized as follows. In section 2 we introduce the Lagrangian, define the counterterms and discuss SSB. Section 3 is devoted to the explanation of our renormalization scheme, while in section 4 we explicitly compute the selfenergies of fermions and scalars at one-loop order. We present an example of a flavour symmetry in section 5 and study how the symmetry teams up with the general renormalization scheme. In section 6 we describe the changes when one has Dirac fermions instead of Majorana fermions. Finally, in section 7 we present the conclusions. Some details which are helpful for reading the paper can be found in the three appendices.

\section{Toy model setup}

In this section, we give the specifics of the investigated model and discuss the generation of masses via SSB. We focus on Majorana fermions. Throughout this paper we always use the sum convention, if not otherwise stated.

\subsection{Bare and renormalized Lagrangian}

The bare Lagrangian is given by

$$
\begin{aligned}
\mathcal{L}_{B}= & i \bar{\chi}_{i L}^{(B)} \gamma^{\mu} \partial_{\mu} \chi_{i L}^{(B)}+\frac{1}{2}\left(\partial_{\mu} \varphi_{a}^{(B)}\right)\left(\partial^{\mu} \varphi_{a}^{(B)}\right) \\
& +\left(\frac{1}{2}\left(Y_{a}^{(B)}\right)_{i j} \chi_{i L}^{(B)} C^{-1} \chi_{j L}^{(B)} \varphi_{a}^{(B)}+\text { H.c. }\right) \\
& -\frac{1}{2}\left(\mu_{B}^{2}\right)_{a b} \varphi_{a}^{(B)} \varphi_{b}^{(B)}-\frac{1}{4} \lambda_{a b c d}^{(B)} \varphi_{a}^{(B)} \varphi_{b}^{(B)} \varphi_{c}^{(B)} \varphi_{d}^{(B)}
\end{aligned}
$$

The charge-conjugation matrix $C$ acts only on the Dirac indices. We assume $n_{\chi}$ chiral Majorana fermion fields $\chi_{i L}^{(B)}$ and $n_{\varphi}$ real scalar fields $\varphi_{a}^{(B)}$. This Lagrangian exhibits the $\mathbb{Z}_{4}$ symmetry

$$
\mathcal{S}: \quad \chi_{L}^{(B)} \rightarrow i \chi_{L}^{(B)}, \quad \varphi^{(B)} \rightarrow-\varphi^{(B)},
$$

\footnotetext{
${ }^{3}$ After SSB, these shifts have to be taken into account everywhere in the Lagrangian where VEVs appear in order to obtain a consistent set of counterterms.
} 
with

$$
\chi_{L}^{(B)}=\left(\begin{array}{c}
\chi_{1 L}^{(B)} \\
\vdots \\
\chi_{n_{\chi} L}^{(B)}
\end{array}\right), \quad \varphi^{(B)}=\left(\begin{array}{c}
\varphi_{1}^{(B)} \\
\vdots \\
\varphi_{n_{\varphi}}^{(B)}
\end{array}\right)
$$

Note that

$$
\left(Y_{a}^{(B)}\right)^{T}=Y_{a}^{(B)} \forall a=1, \ldots, n_{\chi}, \quad\left(\mu_{B}^{2}\right)_{a b}=\left(\mu_{B}^{2}\right)_{b a}
$$

and $\lambda_{a b c d}^{(B)}$ is symmetric in all indices 4

We define the renormalized fields by

$$
\chi_{L}^{(B)}=Z_{\chi}^{(1 / 2)} \chi_{L}, \quad \varphi^{(B)}=Z_{\varphi}^{(1 / 2)} \varphi
$$

where $\chi_{L}$ and $\varphi$ are the vectors of the renormalized fermion and scalar fields, respectively. The quantity $Z_{\chi}^{(1 / 2)}$ is a general complex $n_{\chi} \times n_{\chi}$ matrix, while $Z_{\varphi}^{(1 / 2)}$ is a real but otherwise general $n_{\varphi} \times n_{\varphi}$ matrix. Since we use dimensional regularization with dimension

$$
d=4-\varepsilon
$$

we also introduce an arbitrary mass parameter $\mathcal{M}$ which renders the renormalized Yukawa and quartic coupling constants dimensionless. We split the bare Lagrangian into

$$
\mathcal{L}_{B}=\mathcal{L}+\delta \mathcal{L}
$$

where the renormalized Lagrangian is given by

$$
\begin{aligned}
\mathcal{L}= & i \bar{\chi}_{i L} \gamma^{\mu} \partial_{\mu} \chi_{i L}+\frac{1}{2}\left(\partial_{\mu} \varphi_{a}\right)\left(\partial^{\mu} \varphi_{a}\right) \\
& +\left(\frac{1}{2} \mathcal{M}^{\varepsilon / 2}\left(Y_{a}\right)_{i j} \chi_{i L}^{T} C^{-1} \chi_{j L} \varphi_{a}+\text { H.c. }\right) \\
& -\frac{1}{2} \mu_{a b}^{2} \varphi_{a} \varphi_{b}-\frac{1}{4} \mathcal{M}^{\varepsilon} \lambda_{a b c d} \varphi_{a} \varphi_{b} \varphi_{c} \varphi_{d}
\end{aligned}
$$

and

$$
\begin{aligned}
\delta \mathcal{L}= & i \delta_{i j}^{(\chi)} \bar{\chi}_{i L} \gamma^{\mu} \partial_{\mu} \chi_{j L}+\frac{1}{2} \delta_{a b}^{(\varphi)}\left(\partial_{\mu} \varphi_{a}\right)\left(\partial^{\mu} \varphi_{b}\right) \\
& +\left(\frac{1}{2} \mathcal{M}^{\varepsilon / 2}\left(\delta Y_{a}\right)_{i j} \chi_{i L}^{T} C^{-1} \chi_{j L} \varphi_{a}+\text { H.c. }\right) \\
& -\frac{1}{2} \delta \mu_{a b}^{2} \varphi_{a} \varphi_{b}-\frac{1}{4} \mathcal{M}^{\varepsilon} \delta \lambda_{a b c d} \varphi_{a} \varphi_{b} \varphi_{c} \varphi_{d}
\end{aligned}
$$

contains the counterterms. In $\delta \mathcal{L}$, the counterterms corresponding to the parameters in $\mathcal{L}$ are given by

$$
\begin{aligned}
\mathcal{M}^{\varepsilon / 2} \delta Y_{a} & =\left(Z_{\chi}^{(1 / 2)}\right)^{T} Y_{b}^{(B)} Z_{\chi}^{(1 / 2)}\left(Z_{\varphi}^{(1 / 2)}\right)_{b a}-\mathcal{M}^{\varepsilon / 2} Y_{a} \\
\mathcal{M}^{\varepsilon} \delta \lambda_{a b c d} & =\lambda_{a^{\prime} b^{\prime} c^{\prime} d^{\prime}}^{(B)}\left(Z_{\varphi}^{(1 / 2)}\right)_{a^{\prime} a}\left(Z_{\varphi}^{(1 / 2)}\right)_{b^{\prime} b}\left(Z_{\varphi}^{(1 / 2)}\right)_{c^{\prime} c}\left(Z_{\varphi}^{(1 / 2)}\right)_{d^{\prime} d}-\mathcal{M}^{\varepsilon} \lambda_{a b c d}
\end{aligned}
$$

\footnotetext{
${ }^{4}$ One can show that the number of independent elements of $\lambda_{a b c d}^{(B)}$ is $\left(\begin{array}{c}n_{\varphi}+3 \\ 4\end{array}\right)$.
} 


$$
\delta \mu^{2}=\left(Z_{\varphi}^{(1 / 2)}\right)^{T} \mu_{B}^{2} Z_{\varphi}^{(1 / 2)}-\mu^{2} .
$$

Note that, whenever possible, we use matrix notation, as done in equations (10a) and (10c). Moreover we have defined

$$
\delta^{(\chi)}=\left(Z_{\chi}^{(1 / 2)}\right)^{\dagger} Z_{\chi}^{(1 / 2)}-\mathbb{1}, \quad \delta^{(\varphi)}=\left(Z_{\varphi}^{(1 / 2)}\right)^{T} Z_{\varphi}^{(1 / 2)}-\mathbb{1} .
$$

The renormalized parameters have the same symmetry properties as the unrenormalized ones, i.e.

$$
Y_{a}^{T}=Y_{a} \quad \forall a=1, \ldots, n_{\chi}, \quad \mu_{a b}^{2}=\mu_{b a}^{2}
$$

and $\lambda_{a b c d}$ is symmetric in all indices. The same applies to the corresponding counterterms.

\subsection{Spontaneous symmetry breaking}

We introduce the shift

$$
\varphi_{a}=\mathcal{M}^{-\varepsilon / 2} \bar{v}_{a}+h_{a} \quad \text { with } \quad \bar{v}_{a}=v_{a}+\Delta v_{a} .
$$

For convenience we have split the shift into $v_{a}$ and $\Delta v_{a}$; below we will identify the $v_{a}$ with the tree-level VEVs of the scalar fields $\varphi_{a}$, while the $\Delta v_{a}$ indicate further finite shifts effected by loop corrections. Throughout our calculations, the symbol $\delta$ signifies UV divergent counterterms, while with the symbol $\Delta$ we denote finite shifts. A one-loop discussion of $\Delta v_{a}$ will be presented in section 3. The shift leads to the scalar potential, including counterterms,

$$
\begin{aligned}
V+\delta V-V_{0}= & \mathcal{M}^{-\varepsilon / 2}\left(t_{a}+\Delta t_{a}+\delta \mu_{a b}^{2} \bar{v}_{b}+\delta \lambda_{a b c d} \bar{v}_{b} \bar{v}_{c} \bar{v}_{d}\right) h_{a} \\
& +\frac{1}{2}\left(\left(M_{0}^{2}\right)_{a b}+\left(\Delta M_{0}^{2}\right)_{a b}+\delta \mu_{a b}^{2}+3 \delta \lambda_{a b c d} \bar{v}_{c} \bar{v}_{d}\right) h_{a} h_{b} \\
& +\mathcal{M}^{\varepsilon / 2}\left(\lambda_{a b c d}+\delta \lambda_{a b c d}\right) \bar{v}_{d} h_{a} h_{b} h_{c} \\
& +\frac{1}{4} \mathcal{M}^{\varepsilon}\left(\lambda_{a b c d}+\delta \lambda_{a b c d}\right) h_{a} h_{b} h_{c} h_{d},
\end{aligned}
$$

with $V$ as in equation (9c),

$$
t_{a}=\mu_{a b}^{2} v_{b}+\lambda_{a b c d} v_{b} v_{c} v_{d}, \quad \Delta t_{a}=\mu_{a b}^{2} \bar{v}_{b}+\lambda_{a b c d} \bar{v}_{b} \bar{v}_{c} \bar{v}_{d}-t_{a},
$$

$V_{0}$ being the constant term,

$$
\left(M_{0}^{2}\right)_{a b} \equiv \mu_{a b}^{2}+3 \lambda_{a b c d} v_{c} v_{d} \quad \text { and } \quad\left(\Delta M_{0}^{2}\right)_{a b} \equiv \mu_{a b}^{2}+3 \lambda_{a b c d} \bar{v}_{c} \bar{v}_{d}-\left(M_{0}^{2}\right)_{a b}
$$

The quantities $\Delta t_{a}$ and $\left(\Delta M_{0}^{2}\right)_{a b}$ will become useful when we go beyond the tree level because they will be induced by the shifts $\Delta v_{a}$. We will drop $V_{0}$ in the rest of the paper since it does not alter the dynamics of the theory.

From now on we choose the $v_{a}$ as the tree-level vacuum expectation values (VEVs) of the scalars, i.e. as the values of the $\varphi_{a}$ at the minimum of $V(\varphi)$. Taking the derivative of the scalar potential $V$, we obtain

$$
\frac{\partial V}{\partial \varphi_{a}}=\mu_{a b}^{2} \varphi_{b}+\mathcal{M}^{\varepsilon} \lambda_{a b c d} \varphi_{b} \varphi_{c} \varphi_{d}
$$


Therefore, the conditions that the $v_{a}\left(a=1, \ldots, n_{\varphi}\right)$ correspond to a stationary point of $V$ are given by

$$
t_{a}=0 \quad \text { for } \quad a=1, \ldots, n_{\varphi} .
$$

$\mathrm{SSB}$ occurs if the minimum $\varphi_{1}=v_{1}, \ldots, \varphi_{n_{\varphi}}=v_{n_{\varphi}}$ of $V$ is non-trivial, i.e. different from $v_{1}=\cdots=v_{n_{\varphi}}=0$. In any case, whether there is SSB or not, $M_{0}^{2}$ of equation (16) is the tree level mass matrix of the scalars.

The mass matrix of the fermions is given by

$$
m_{0}=\sum_{a=1}^{n_{\varphi}} v_{a} Y_{a} .
$$

The subscript 0 in $m_{0}$ and $M_{0}^{2}$ indicates tree level mass matrices. The tree-level mass matrices and fermions and scalars are diagonalized by

$$
\begin{aligned}
U_{0}^{T} m_{0} U_{0} & =\hat{m}_{0} \equiv \operatorname{diag}\left(m_{01}, \ldots, m_{0 n_{\chi}}\right), \\
W_{0}^{T} M_{0}^{2} W_{0} & =\hat{M}_{0}^{2} \equiv \operatorname{diag}\left(M_{01}^{2}, \ldots, M_{0 n_{\varphi}}^{2}\right),
\end{aligned}
$$

where $U_{0}$ is unitary [15] and $W_{0}$ is orthogonal.

The diagonalization matrices $U_{0}$ and $W_{0}$ allow us to introduce mass eigenfields $\hat{\chi}_{j L}$ and $\hat{h}_{a}$ via

$$
\chi_{i L}=\left(U_{0}\right)_{i j} \hat{\chi}_{j L} \quad \text { and } \quad h_{a}=\left(W_{0}\right)_{a b} \hat{h}_{b},
$$

respectively. Rewriting the Lagrangian in terms of the mass eigenfields amounts to the replacements

$$
\begin{aligned}
\delta^{(\chi)} & \rightarrow \hat{\delta}^{(\chi)}=U_{0}^{\dagger} \delta^{(\chi)} U_{0} \\
Y_{a} & \rightarrow \hat{Y}_{a}=\left(U_{0}^{T} Y_{b} U_{0}\right)\left(W_{0}\right)_{b a} \\
\delta^{(\varphi)} & \rightarrow \hat{\delta}^{(\varphi)}=W_{0}^{T} \delta^{(\varphi)} W_{0} \\
v_{a} & \rightarrow \hat{v}_{a}=\left(W_{0}\right)_{b a} v_{b} \\
t_{a} & \rightarrow \hat{t}_{a}=\left(W_{0}\right)_{b a} t_{b} \\
\mu^{2} & \rightarrow \hat{\mu}^{2}=W_{0}^{T} \mu^{2} W_{0} \\
\lambda_{a b c d} & \rightarrow \hat{\lambda}_{a b c d}=\lambda_{a^{\prime} b^{\prime} c^{\prime} d^{\prime}}\left(W_{0}\right)_{a^{\prime} a}\left(W_{0}\right)_{b^{\prime} b}\left(W_{0}\right)_{c^{\prime} c}\left(W_{0}\right)_{d^{\prime} d}
\end{aligned}
$$

such that the form of the Lagrangian is preserved. Therefore, without loss of generality we assume that we are in the mass bases of fermions and scalars, when we perform the one-loop computation of the selfenergies. Note that $\hat{\bar{v}}_{a}$ and $\Delta \hat{v}_{a}$ are defined analogously to $\hat{v}_{a}$.

In the mass basis it is useful to rewrite the Yukawa interaction as

$$
\mathcal{L}_{Y}=-\frac{1}{2} \overline{\hat{\chi}}\left(\hat{Y}_{a} \gamma_{L}+\hat{Y}_{a}^{*} \gamma_{R}\right) \hat{\chi}\left(\mathcal{M}^{\varepsilon / 2} \hat{h}_{a}+\hat{\bar{v}}_{a}\right)
$$

with

$$
\gamma_{L}=\frac{\mathbb{1}-\gamma_{5}}{2}, \quad \gamma_{R}=\frac{\mathbb{1}+\gamma_{5}}{2}, \quad \hat{\chi}=\left(\begin{array}{c}
\hat{\chi}_{1} \\
\vdots \\
\hat{\chi}_{n_{\chi}}
\end{array}\right) \quad \text { and } \quad \hat{\chi}_{i}=\hat{\chi}_{i L}+\left(\hat{\chi}_{i L}\right)^{c},
$$

where the superscript $c$ indicates charge conjugation. 


\section{Renormalization}

General outline: Our objective is to describe the general renormalization procedure and to work out a prescription for the computation of the one-loop contribution to the physical fermion and scalar masses. For this purpose we have to compute the selfenergies. Clearly, the manner in which the selfenergies - and thus the quantities we aim at - depend on the parameters of our toy model is renormalization-scheme-dependent. It is, therefore, expedient to clearly expound the scheme we want to use and how we plan to reach our goal.

We proceed in three steps:

i. $\overline{\mathrm{MS}}$ renormalization for the determination of $\delta \hat{Y}_{a}, \delta \hat{\lambda}_{a b c d}, \delta \hat{\mu}_{a b}^{2}, \hat{\delta}^{(\chi)}$ and $\hat{\delta}^{(\varphi)}$.

ii. Finite shifts $\Delta \hat{v}_{a}$ such that the scalar one-point functions of the $\hat{h}_{a}$ are zero. These two steps allow us to compute renormalized one-loop selfenergies $\Sigma(p)$ and $\Pi\left(p^{2}\right)$ for fermions and scalars, respectively.

iii. Finite field strength renormalization in order to switch from the $\overline{\mathrm{MS}}$ selfenergies $\Sigma(p)$ and $\Pi\left(p^{2}\right)$ to on-shell selfenergies $5 \widetilde{\Sigma}(p)$ and $\widetilde{\Pi}\left(p^{2}\right)$.

Several remarks are in order to concretize this outline. $\overline{\mathrm{MS}}$ renormalization, i.e. subtraction of terms proportional to the constant

$$
c_{\infty}=\frac{2}{\varepsilon}-\gamma+\ln (4 \pi)
$$

where $\gamma$ is the Euler-Mascheroni constant, is realized in the following way:

(a) $\delta \hat{\lambda}_{a b c d}$ is determined from the quartic scalar coupling,

(b) $\delta \hat{Y}_{a}$ is obtained from the Yukawa vertex,

(c) $\delta \hat{\mu}_{a b}^{2}$ removes $c_{\infty}$ from the $p^{2}$-independent part of the scalar selfenergy,

(d) $\hat{\delta}^{(\chi)}$ and $\hat{\delta}^{(\varphi)}$ are determined from the momentum-dependent parts of the respective selfenergies.

With the prescriptions (a)-(d) above, all correlation functions and all physical quantities computed in our toy model must be finite. This applies in particular to the selfenergies.

Fermion selfenergy: Let us first consider the renormalized fermion selfenergy $\Sigma(p)$, defined via the inverse propagator matrix

$$
S^{-1}(p)=\not p-\hat{m}_{0}-\Sigma(p)
$$

\footnotetext{
${ }^{5}$ Note that here the term on-shell refers to field strength renormalization only. We have no mass counterterms, because in our approach masses are derived quantities and, therefore, functions of the parameters of the model - see the discussion at the end of this section.
} 
where $\Sigma(p)$ has the chiral structure

$$
\Sigma(p)=\not p\left(\Sigma_{L}^{(A)}\left(p^{2}\right) \gamma_{L}+\Sigma_{R}^{(A)}\left(p^{2}\right) \gamma_{R}\right)+\Sigma_{L}^{(B)}\left(p^{2}\right) \gamma_{L}+\Sigma_{R}^{(B)}\left(p^{2}\right) \gamma_{R}
$$

For the relationships between $\Sigma_{L}^{(A)}$ and $\Sigma_{R}^{(A)}$ and between $\Sigma_{L}^{(B)}$ and $\Sigma_{R}^{(B)}$ in the case of Dirac and Majorana fermions we refer the reader to appendix A. At one-loop order, $\Sigma(p)$ has the terms

$$
\begin{aligned}
\Sigma(p)= & \Sigma^{1 \text {-loop }}(p)-\not p\left[\hat{\delta}^{(\chi)} \gamma_{L}+\left(\hat{\delta}^{(\chi)}\right)^{*} \gamma_{R}\right] \\
& +\hat{v}_{a}\left[\delta \hat{Y}_{a} \gamma_{L}+\left(\delta \hat{Y}_{a}\right)^{*} \gamma_{R}\right]+\Delta \hat{v}_{a}\left[\hat{Y}_{a} \gamma_{L}+\hat{Y}_{a}^{*} \gamma_{R}\right]
\end{aligned}
$$

where $\Sigma^{1 \text {-loop }}$ corresponds to the diagram of figure 1, Since $\delta \hat{Y}_{a}$ is already determined by the Yukawa vertex, the corresponding term in $\Sigma(p)$ must automatically make $\Sigma_{L, R}^{(B)}$ in equation (28) finite. As for $\Sigma_{L, R}^{(A)}$ in $\Sigma(p)$, we note that these matrices are hermitian-see also appendix A, therefore, the counterterms with the hermitian matrix $\hat{\delta}^{(\chi)}$ suffice for finiteness. The last term in equation (28) is induced by the finite VEV shifts.

Scalar selfenergy: Now we address the inverse scalar propagator matrix

$$
\Delta^{-1}\left(p^{2}\right)=p^{2}-\hat{M}_{0}^{2}-\Pi\left(p^{2}\right) .
$$

The scalar selfenergy $\Pi\left(p^{2}\right)$ has the structure

$$
\Pi_{a b}\left(p^{2}\right)=\Pi_{a b}^{1-\text { loop }}\left(p^{2}\right)-\hat{\delta}_{a b}^{(\varphi)} p^{2}+\delta \hat{\mu}_{a b}^{2}+3 \delta \hat{\lambda}_{a b c d} \hat{v}_{c} \hat{v}_{d}+6 \hat{\lambda}_{a b c d} \hat{v}_{c} \Delta \hat{v}_{d}
$$

at one-loop order. With an argument analogous to the fermionic case we find that the symmetric matrix $\hat{\delta}^{(\varphi)}$ suffices for making the derivative of $\Pi\left(p^{2}\right)$ finite. According to our renormalization prescription, $\delta \hat{\lambda}_{a b c d} \hat{v}_{c} \hat{v}_{d}$ is already fixed, but we have $\delta \hat{\mu}_{a b}^{2}$ at our disposal to cancel the infinity in the $p^{2}$-independent term in $\Pi\left(p^{2}\right)$. The last term in the scalar selfenergy, equation (30), stems from the finite mass corrections $\Delta M_{0}^{2}$ - see equation (14b) - expressed in terms of the finite VEV shifts induced by tadpole contributions.

Another commonly used approach for the renormalization of $\hat{\mu}^{2}, e . g$. in [12], is to express its diagonal entries via the tadpole parameters $\hat{t}_{a}$ as of equation (15), resulting in renormalization conditions more closely related to physical observables. However, there are simply not enough tadpole parameters available to replace all parameters in the $n_{\varphi} \times n_{\varphi}$ symmetric matrix $\hat{\mu}^{2}$ and we have two main reasons for dismissing this choice in our case. One is that expressing $\hat{\mu}_{a a}^{2}$ in terms of the tadpole parameters involves the inverses of the VEVs $\hat{v}_{a}$. In the general case, some of these can be zero, leading to ill-defined expressions for $\delta \hat{\mu}_{a a}^{2}$. The other one is that the diagonal and off-diagonal entries of $\hat{\mu}^{2}$ can be treated on an equal footing in our approach, leading to a more compact description.

One-point function: These shifts derive from the linear term in the scalar potential. For simplicity we stick to the lowest non-trivial order, where it is given by

$$
\mathcal{M}^{-\varepsilon / 2}\left(\hat{t}_{a}+\Delta \hat{t}_{a}+\delta \hat{\mu}_{a b}^{2} \hat{v}_{b}+\delta \hat{\lambda}_{a b c d} \hat{v}_{b} \hat{v}_{c} \hat{v}_{d}\right) \hat{h}_{a}
$$


Diagrammatically, the one-point function pertaining to $\hat{h}_{a}$ has the contributions 6

$$
\begin{aligned}
& i+\underset{1}{i}+\underset{1}{x} \\
& =\mathcal{M}^{-\varepsilon / 2} \frac{i}{-M_{0 a}^{2}} \times(-i)\left(\hat{t}_{a}+T_{a}+\Delta \hat{t}_{a}+\delta \hat{\mu}_{a b}^{2} \hat{v}_{b}+\delta \hat{\lambda}_{a b c d} \hat{v}_{b} \hat{v}_{c} \hat{v}_{d}\right)=0,
\end{aligned}
$$

where $i /\left(-M_{o a}^{2}\right)$ is the external scalar propagator at zero momentum. The requirement that the one-point function is zero is identical with the requirement that the VEV of $\hat{h}_{a}$ is zero. The first diagram in equation (32) represents the scalar tree-level one-point function corresponding to $\hat{t}_{a}$, which vanishes identically due to equation (18); we have included it only for illustrative purposes. The second diagram, which represents the one-loop tadpole contributions, corresponds to $T_{a}$. The third diagram represents the sum of $\Delta \hat{t}_{a}$ and the two counterterm contributions. We can decompose $T_{a}$ into an infinite and a finite part, i.e.

$$
T_{a}=\left(T_{\infty}\right)_{a}+\left(T_{\text {fin }}\right)_{a} .
$$

Since with the imposition of conditions (a)-(d) the theory becomes finite, in equation (31) we necessarily have

$$
\delta \hat{\mu}_{a b}^{2} \hat{v}_{b}+\delta \hat{\lambda}_{a b c d} \hat{v}_{b} \hat{v}_{c} \hat{v}_{d}+\left(T_{\infty}\right)_{a}=0 .
$$

An explicit check of this relation is presented in section 4.3. Moreover, we translate the finite tadpole contributions $\left(T_{\mathrm{fin}}\right)_{a}$ to shifts of the VEVs $\Delta \hat{v}_{b}$, similar to the approach of [9]. At one-loop order this is effected by

$$
\Delta \hat{t}_{a}=\hat{\mu}_{a b}^{2} \Delta \hat{v}_{b}+3 \hat{\lambda}_{a b c d} \hat{v}_{c} \hat{v}_{d} \Delta \hat{v}_{b}=\left(\hat{M}_{0}^{2}\right)_{a b} \Delta \hat{v}_{b}
$$

where we have used equation (15). Therefore, equation (32) leads to the finite shift

$$
\Delta \hat{v}_{a}=-\left(\hat{M}_{0}^{2}\right)_{a b}^{-1}\left(T_{\text {fin }}\right)_{b}
$$

Note that these finite shifts eventually contribute to the finite mass corrections because they contribute to the two-point functions of the fermions and scalars - see equations (28) and (30), respectively. Further clarifications concerning the VEV shifts $\Delta v_{a}$ are found in appendix B.

Pole masses and finite field strength renormalization: It remains to perform a finite field strength renormalization in order to transform the one-loop selfenergies $\Sigma(p)$ and $\Pi\left(p^{2}\right)$ to on-shell selfenergies $\widetilde{\Sigma}(p)$ and $\widetilde{\Pi}\left(p^{2}\right)$, respectively. Immediately the question arises why we cannot use the $Z_{\chi}^{(1 / 2)}$ and $Z_{\varphi}^{(1 / 2)}$ defined in section 2.1 for this purpose. Note that we have incorporated these matrices into $\delta Y_{a}$ and $\delta \lambda_{a b c d}$ at the respective interaction vertices. Therefore, in $\delta \mathcal{L}$ the field strength renormalization matrices $Z_{\chi}^{(1 / 2)}$ and $Z_{\varphi}^{(1 / 2)}$ occur solely in the hermitian matrix $\delta^{(\chi)}$ and the symmetric matrix $\delta^{(\varphi)}$, respectively.

\footnotetext{
${ }^{6}$ We stress again that we do not introduce tadpole counterterms.
} 
Obviously, the latter matrices have fewer parameters than the original ones and it is impossible to perform on-shell renormalization with $\delta^{(\chi)}$ for more than one fermion field and with $\delta^{(\varphi)}$ for more than one scalar field. What happens if we do not incorporate $Z_{\chi}^{(1 / 2)}$ and $Z_{\varphi}^{(1 / 2)}$ into the Yukawa and quartic couplings, respectively? Let us consider the Yukawa interaction for definiteness and denote by $\check{\delta} Y_{a}$ the Yukawa counterterm where $Z_{\chi}^{(1 / 2)}$ is not incorporated. Obviously, the relation between $\delta Y_{a}$ and $\check{\delta} Y_{a}$ is given by

$$
\delta Y_{a}=\left(Z_{\chi}^{(1 / 2)}\right)^{T}\left(Y_{b}+\check{\delta} Y_{b}\right) Z_{\chi}^{(1 / 2)}\left(Z_{\varphi}^{(1 / 2)}\right)_{b a}-Y_{a}
$$

Actually, the quantity that is determined by the $\overline{\mathrm{MS}}$ Yukawa vertex renormalization is $\delta Y_{a}$ and not $\check{\delta} Y_{a}$. Moreover, since we generate mass terms by SSB, the fermion mass term is induced by the shift of equation (13) and has the form

$$
\frac{1}{2} \chi_{L}^{T} C^{-1} \delta Y_{a} \bar{v}_{a} \chi_{L}+\frac{1}{2} \chi_{L}^{T} C^{-1} Y_{a} \bar{v}_{a} \chi_{L}+\text { H.c. }
$$

Thus it is clearly the same $\delta Y_{a}$ that occurs in both the mass term and the vertex renormalization. Consequently, with the counterterms of the unbroken theory we always end up with $\delta Y_{a}$ and $\delta^{(\chi)}$ as independent quantities and we can in general not perform on-shell renormalization. Therefore, we need, in addition to $Z_{\chi}^{(1 / 2)}$ and $Z_{\varphi}^{(1 / 2)}$, finite field strength renormalization matrices $\stackrel{\circ}{Z}_{\chi}^{(1 / 2)}$ and $\stackrel{\circ}{Z}_{h}^{(1 / 2)}$ for fermions and bosons, respectively, inserted into the broken Lagrangian, in order to perform on-shell renormalization. In this way, the $\sqrt{Z}$-factors of the external lines in the LSZ formalism are exactly one [5].

We denote the one-loop contributions to $\stackrel{\circ}{Z}_{\chi}^{(1 / 2)}$ and $\stackrel{\circ}{Z}_{h}^{(1 / 2)}$ by $\frac{1}{2} \stackrel{\circ}{z}_{\chi}$ and $\frac{1}{2} \stackrel{\circ}{z}_{h}$, respectively. For the details of the computation and the results for these quantities we refer the reader to appendix A. Here we only state the masses [16, 17]

$$
\begin{aligned}
m_{i} & =m_{0 i}+m_{0 i}\left(\Sigma_{L}^{(A)}\right)_{i i}\left(m_{0 i}^{2}\right)+\operatorname{Re}\left(\Sigma_{L}^{(B)}\right)_{i i}\left(m_{0 i}^{2}\right), \\
M_{a}^{2} & =M_{0 a}^{2}+\Pi_{a a}\left(M_{0 a}^{2}\right)
\end{aligned}
$$

at one-loop order. There is no summation in these two formulas over equal indices.

Eventually we remark that one could decompose $\stackrel{\circ}{z}$ into a hermitian and an antihermitian matrix. One could be tempted to conceive the antihermitian part as a correction to the tree-level diagonalization matrix $U_{0}$. However, we think that in our simple model such a decomposition has no physical meaning; in essence, we have no PMNS mixing matrix at disposal where it could become physical. Of course, a similar remark applies to $\stackrel{\circ}{z}_{h}$ - see also [18] for a recent discussion in the context of the two-Higgs-doublet model.

\section{Renormalization at the one-loop level}

In this section we concretize, at the one-loop level, the renormalization procedure introduced in the previous section. For the relevant integrals needed for these computations see appendix C. 


\subsection{One-loop results for selfenergies and tadpoles}

Here we display the results for the one-loop contributions $\Sigma^{1 \text {-loop }}(p)$ and $\Pi_{a b}^{1-l o o p}\left(p^{2}\right)$ to the fermion and scalar selfenergies, respectively, and also for the one-loop tadpole expression $T_{a}$.

Fermion selfenergy: The only direct one-loop contribution to the fermionic self-energy is given by the diagram of figure 1. Then, the definitions

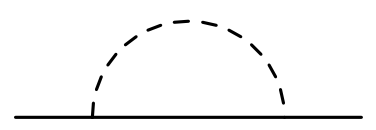

Figure 1: One-loop fermion selfenergy diagram.

$$
\begin{gathered}
\Delta_{a, k}=x M_{0 a}^{2}+(1-x) m_{0 k}^{2}-x(1-x) p^{2} \\
D_{a, k}=\int_{0}^{1} \mathrm{~d} x x \ln \frac{\Delta_{a, k}}{\mathcal{M}^{2}}, \quad E_{a, k}=\int_{0}^{1} \mathrm{~d} x \ln \frac{\Delta_{a, k}}{\mathcal{M}^{2}}
\end{gathered}
$$

and

$$
\hat{D}_{a}=\operatorname{diag}\left(D_{a, 1}, \ldots, D_{a, n_{\chi}}\right), \quad \hat{E}_{a}=\operatorname{diag}\left(E_{a, 1}, \ldots, E_{a, n_{\chi}}\right)
$$

allow us to write the one-loop contribution to the fermionic selfenergy as

$$
\begin{aligned}
\Sigma^{1 \text {-loop }}= & \frac{1}{16 \pi^{2}}\left\{\not p \gamma_{L}\left[-\frac{1}{2} c_{\infty} \hat{Y}_{a}^{*} \hat{Y}_{a}+\hat{Y}_{a}^{*} \hat{D}_{a} \hat{Y}_{a}\right]\right. \\
& +\not p \gamma_{R}\left[-\frac{1}{2} c_{\infty} \hat{Y}_{a} \hat{Y}_{a}^{*}+\hat{Y}_{a} \hat{D}_{a} \hat{Y}_{a}^{*}\right] \\
& +\gamma_{L}\left[-c_{\infty} \hat{Y}_{a} \hat{m}_{0} \hat{Y}_{a}+\hat{Y}_{a} \hat{m}_{0} \hat{E}_{a} \hat{Y}_{a}\right] \\
& \left.+\gamma_{R}\left[-c_{\infty} \hat{Y}_{a}^{*} \hat{m}_{0} \hat{Y}_{a}^{*}+\hat{Y}_{a}^{*} \hat{m}_{0} \hat{E}_{a} \hat{Y}_{a}^{*}\right]\right\} .
\end{aligned}
$$

Scalar selfenergy: In the following, the superscripts $(a),(b),(c)$ refer to the Feynman diagrams of figure 2. Thus the selfenergy has the contributions

$$
\Pi_{a b}^{1-\text { loop }}\left(p^{2}\right)=\Pi_{a b}^{(a)}\left(p^{2}\right)+\Pi_{a b}^{(b)}\left(p^{2}\right)+\Pi_{a b}^{(c)}\left(p^{2}\right) .
$$

We define

$$
\Delta_{i j}=x m_{0 i}^{2}+(1-x) m_{0 j}^{2}-x(1-x) p^{2} \quad \text { and } \quad \tilde{\Delta}_{r s}=x M_{0 r}^{2}+(1-x) M_{0 s}^{2}-x(1-x) p^{2} .
$$

With these definitions we obtain

$$
\Pi_{a b}^{(a)}\left(p^{2}\right)=\frac{1}{16 \pi^{2}}\left\{c_{\infty} \operatorname{Tr}\left[\hat{Y}_{a} \hat{m}_{0} \hat{Y}_{b} \hat{m}_{0}+\hat{Y}_{a}^{*} \hat{m}_{0} \hat{Y}_{b}^{*} \hat{m}_{0}+2 \hat{Y}_{a} \hat{Y}_{b}^{*} \hat{m}_{0}^{2}+2 \hat{Y}_{a}^{*} \hat{Y}_{b} \hat{m}_{0}^{2}\right]\right.
$$




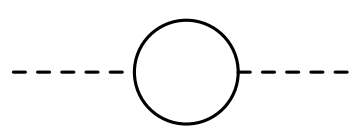

(a)

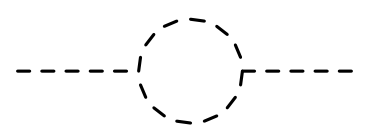

(b)

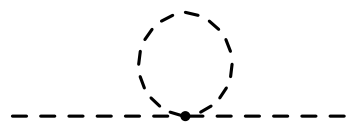

(c)

Figure 2: The Feynman diagrams of the one-loop contributions to the scalar selfenergy.

$$
\begin{aligned}
& -\frac{1}{2} c_{\infty} \operatorname{Tr}\left[\hat{Y}_{a} \hat{Y}_{b}^{*}+\hat{Y}_{a}^{*} \hat{Y}_{b}\right] p^{2} \\
& +\operatorname{Tr}\left[\left(\hat{Y}_{a} \hat{Y}_{b}^{*}+\hat{Y}_{a}^{*} \hat{Y}_{b}\right)\left(\hat{m}_{0}^{2}-\frac{1}{6} p^{2}\right)\right] \\
& -\int_{0}^{1} \mathrm{~d} x\left[\left(\left(\hat{Y}_{a}\right)_{i j}\left(\hat{Y}_{b}\right)_{j i}^{*}+\left(\hat{Y}_{a}\right)_{i j}^{*}\left(\hat{Y}_{b}\right)_{j i}\right)\left(2 \Delta_{i j}-x(1-x) p^{2}\right)\right. \\
& \left.\left.+\left(\hat{Y}_{a}\right)_{i j} m_{0 j}\left(\hat{Y}_{b}\right)_{j i} m_{0 i}+\left(\hat{Y}_{a}\right)_{i j}^{*} m_{0 j}\left(\hat{Y}_{b}\right)_{j i}^{*} m_{0 i}\right] \ln \frac{\Delta_{i j}}{\mathcal{M}^{2}}\right\}, \\
\Pi_{a b}^{(b)}\left(p^{2}\right)= & -\frac{18}{16 \pi^{2}} \hat{\lambda}_{a c r s} \hat{v}_{c} \hat{\lambda}_{b d r s} \hat{v}_{d}\left(c_{\infty}-\int_{0}^{1} \mathrm{~d} x \ln \frac{\tilde{\Delta}_{r s}}{\mathcal{M}^{2}}\right) \\
\Pi_{a b}^{(c)}\left(p^{2}\right)= & -\frac{3}{16 \pi^{2}} \hat{\lambda}_{a b r r} M_{0 r}^{2}\left(c_{\infty}+1-\ln \frac{M_{0 r}^{2}}{\mathcal{M}^{2}}\right) .
\end{aligned}
$$

For the following discussion, it is useful to introduce a separate notation for the divergent $p^{2}$-independent parts of $\Pi_{a b}^{1-\text { loop }}$ :

$$
\begin{aligned}
\left(\Pi_{\infty}^{(a)}\right)_{a b} & =\frac{1}{16 \pi^{2}} c_{\infty} \operatorname{Tr}\left[\hat{Y}_{a} \hat{m}_{0} \hat{Y}_{b} \hat{m}_{0}+\hat{Y}_{a}^{*} \hat{m}_{0} \hat{Y}_{b}^{*} \hat{m}_{0}+2 \hat{Y}_{a} \hat{Y}_{b}^{*} \hat{m}_{0}^{2}+2 \hat{Y}_{a}^{*} \hat{Y}_{b} \hat{m}_{0}^{2}\right] \\
\left(\Pi_{\infty}^{(b)}\right)_{a b} & =-\frac{18}{16 \pi^{2}} c_{\infty} \hat{\lambda}_{a c r s} \hat{v}_{c} \hat{\lambda}_{b d r s} \hat{v}_{d} \\
\left(\Pi_{\infty}^{(c)}\right)_{a b} & =-\frac{3}{16 \pi^{2}} c_{\infty} \hat{\lambda}_{a b r r} M_{0 r}^{2}
\end{aligned}
$$

Tadpoles: There are two one-loop tadpole contributions to the scalar one-point function, namely

$$
\bigcup_{1}+i_{1}^{1-1}=\mathcal{M}^{-\varepsilon / 2} \frac{i}{-M_{0 a}^{2}} \times(-i)\left(T_{a}^{(\chi)}+T_{a}^{(h)}\right) .
$$

We find the following result for tadpole terms:

$$
\begin{aligned}
T_{a}^{(\chi)} & =\frac{1}{16 \pi^{2}} \operatorname{Tr}\left[\left(\hat{Y}_{a} \hat{m}_{0}^{3}+\hat{Y}_{a}^{*} \hat{m}_{0}^{3}\right)\left(c_{\infty}+1-\ln \frac{\hat{m}_{0}^{2}}{\mathcal{M}^{2}}\right)\right], \\
T_{a}^{(h)} & =-\frac{3}{16 \pi^{2}} \hat{\lambda}_{a b r r} \hat{v}_{b} M_{0 r}^{2}\left(c_{\infty}+1-\ln \frac{M_{0 r}^{2}}{\mathcal{M}^{2}}\right) .
\end{aligned}
$$

We denote the divergences in the tadpole expressions by $\left(T_{\infty}^{(\chi)}\right)_{a}$ and $\left(T_{\infty}^{(h)}\right)_{a}$. 


\subsection{Determination of the counterterms}

Counterterms of Yukawa and quartic scalar couplings: Using $\overline{\mathrm{MS}}$ renormalization, it is straightforward to compute these counterterms. For the Yukawa couplings we obtain

$$
\delta \hat{Y}_{a}=\frac{1}{16 \pi^{2}} c_{\infty} \hat{Y}_{b} \hat{Y}_{a}^{*} \hat{Y}_{b}
$$

The $\delta \hat{\lambda}_{a b c d}$ can be split into

$$
\delta \hat{\lambda}_{a b c d}=\delta \hat{\lambda}_{a b c d}^{(\chi)}+\delta \hat{\lambda}_{a b c d}^{(\varphi)}
$$

generated by fermions and scalars, respectively, in the loop. The first case yields

$$
\begin{aligned}
\delta \hat{\lambda}_{a b c d}^{(\chi)}= & -\frac{1}{3} \times \frac{1}{16 \pi^{2}} c_{\infty} \operatorname{Tr}\left[\hat{Y}_{a} \hat{Y}_{b}^{*} \hat{Y}_{c} \hat{Y}_{d}^{*}+\hat{Y}_{a} \hat{Y}_{c}^{*} \hat{Y}_{d} \hat{Y}_{b}^{*}+\hat{Y}_{a} \hat{Y}_{d}^{*} \hat{Y}_{b} \hat{Y}_{c}^{*}\right. \\
& \left.+\hat{Y}_{a}^{*} \hat{Y}_{b} \hat{Y}_{c}^{*} \hat{Y}_{d}+\hat{Y}_{a}^{*} \hat{Y}_{c} \hat{Y}_{d}^{*} \hat{Y}_{b}+\hat{Y}_{a}^{*} \hat{Y}_{d} \hat{Y}_{b}^{*} \hat{Y}_{c}\right]
\end{aligned}
$$

In this formula we have taken into account that the Yukawa coupling matrices are symmetric. The scalar contribution is

$$
\delta \hat{\lambda}_{a b c d}^{(\varphi)}=\frac{3}{16 \pi^{2}} c_{\infty}\left(\hat{\lambda}_{a b r s} \hat{\lambda}_{r s c d}+\hat{\lambda}_{a d r s} \hat{\lambda}_{r s b c}+\hat{\lambda}_{a c r s} \hat{\lambda}_{r s b d}\right)
$$

Counterterms pertaining to field strength renormalization: Cancellation of the divergence in equation (44b) determines $\hat{\delta}^{(\chi)}$ as

$$
\hat{\delta}^{(\chi)}=-\frac{1}{2} \times \frac{1}{16 \pi^{2}} c_{\infty} \hat{Y}_{a}^{*} \hat{Y}_{a}
$$

Considering the scalar selfenergy, we find that only diagram (a) of figure 2 has a divergence proportional to $p^{2}$. Therefore, we obtain from equation (47a)

$$
\hat{\delta}_{a b}^{(\varphi)}=-\frac{1}{2} \times \frac{1}{16 \pi^{2}} c_{\infty} \operatorname{Tr}\left[\hat{Y}_{a} \hat{Y}_{b}^{*}+\hat{Y}_{a}^{*} \hat{Y}_{b}\right]
$$

Counterterm pertaining to $\hat{\mu}_{a b}^{2}$ : The counterterm $\delta \hat{\mu}_{a b}^{2}$ has to be determined by the cancellations of the divergences of equations (48b) and (48c). Thus we demand

$$
\begin{aligned}
0 & =\delta \hat{\mu}_{a b}^{2}+3 \delta \hat{\lambda}_{a b c d}^{(\varphi)} \hat{v}_{c} \hat{v}_{d}+\left(\Pi_{\infty}^{(b)}\right)_{a b}+\left(\Pi_{\infty}^{(c)}\right)_{a b} \\
& =\delta \hat{\mu}_{a b}^{2}+\frac{3}{16 \pi^{2}} c_{\infty}\left[3 \hat{\lambda}_{a b r s} \hat{\lambda}_{r s c d} \hat{v}_{c} \hat{v}_{d}-\hat{\lambda}_{a b r r} M_{0 r}^{2}\right] \\
& =\delta \hat{\mu}_{a b}^{2}+\frac{3}{16 \pi^{2}} c_{\infty}\left[\hat{\lambda}_{a b r s}\left(\hat{\mu}_{r s}^{2}+3 \hat{\lambda}_{r s c d} \hat{v}_{c} \hat{v}_{d}-\hat{\mu}_{r s}^{2}\right)-\hat{\lambda}_{a b r r} M_{0 r}^{2}\right] \\
& =\delta \hat{\mu}_{a b}^{2}-\frac{3}{16 \pi^{2}} c_{\infty} \hat{\lambda}_{a b r s} \hat{\mu}_{r s}^{2} .
\end{aligned}
$$

Therefore, $\delta \hat{\mu}_{a b}^{2}$ is fixed as

$$
\delta \hat{\mu}_{a b}^{2}=\frac{3}{16 \pi^{2}} c_{\infty} \hat{\lambda}_{a b r s} \hat{\mu}_{r s}^{2}
$$




\subsection{Cancellation of divergences}

Having fixed all available counterterms, the remaining UV divergences in the selfenergies and tadpoles have to drop out. This is what we want to show in this subsection.

Fermion selfenergy: With $\delta \hat{Y}_{a}$ of equation (52) and

$$
\hat{v}_{a} \delta \hat{Y}_{a}=\frac{1}{16 \pi^{2}} c_{\infty} \hat{Y}_{b} \hat{m}_{0} \hat{Y}_{b}
$$

we find that $\Sigma$ is finite without any mass renormalization, as it has to be.

Scalar selfenergy: We have already treated the divergences (48b) and (48c), but there is still the divergence of equation (48a). However, it is easy to see that its cancellation in the selfenergy (30) is simply effected by

$$
\left(\Pi_{\infty}^{(a)}\right)_{a b}+3 \delta \hat{\lambda}_{a b c d}^{(\chi)} \hat{v}_{c} \hat{v}_{d}=0
$$

Tadpoles: It remains to verify equation (34). First we consider the result of the fermionic tadpole in equation (50). Contracting the counterterm $\delta \hat{\lambda}_{\text {abcd }}^{(\chi)}$ of equation (54) with the VEVs and adding to it $\left(T_{\infty}^{(\chi)}\right)_{a}$ yields

$$
\left(T_{\infty}^{(\chi)}\right)_{a}+\delta \hat{\lambda}_{a b c d}^{(\chi)} \hat{v}_{b} \hat{v}_{c} \hat{v}_{d}=\left(T_{\infty}^{(\chi)}\right)_{a}-\frac{1}{16 \pi^{2}} c_{\infty} \operatorname{Tr}\left[\left(\hat{Y}_{a} \hat{m}_{0}^{3}+\hat{Y}_{a}^{*} \hat{m}_{0}^{3}\right)\right]=0
$$

Similarly, using equations (55) and (59), the scalar tadpole contribution of equation (51) is found to be finite via

$$
\begin{aligned}
& \left(T_{\infty}^{(h)}\right)_{a}+\delta \hat{\mu}_{a b}^{2} \hat{v}_{b}+\delta \hat{\lambda}_{a b c d}^{(\varphi)} \hat{v}_{b} \hat{v}_{c} \hat{v}_{d} \\
& =\left(T_{\infty}^{(h)}\right)_{a}+\frac{3}{16 \pi^{2}} c_{\infty}\left(\hat{\lambda}_{a b r s} \hat{\mu}_{r s}^{2} \hat{v}_{b}+3 \hat{\lambda}_{a b r s} \hat{\lambda}_{r s c d} \hat{v}_{b} \hat{v}_{c} \hat{v}_{d}\right) \\
& =\left(T_{\infty}^{(h)}\right)_{a}+\frac{3}{16 \pi^{2}} c_{\infty} \hat{\lambda}_{a b r r} \hat{v}_{b} M_{0 r}^{2}=0 .
\end{aligned}
$$

\subsection{Counterterms and UV divergences in a general basis}

The results for the selfenergies and counterterms shown in the previous sections are given in the mass bases. However, for a check of the cancellation of divergences it might be advantageous to have the divergences in a general basis. Such expressions can be obtained by using the parameter transformations (22).

As an example, let us do this transformation in the case of $\hat{\delta}^{(\chi)}$ of equation (56), where one has to apply

$$
\begin{aligned}
\delta^{(\chi)} & =U_{0} \hat{\delta}^{(\chi)} U_{0}^{\dagger} \\
& =-\frac{1}{2} \times \frac{1}{16 \pi^{2}} c_{\infty} U_{0} \hat{Y}_{a}^{*} \hat{Y}_{a} U_{0}^{\dagger} \\
& =-\frac{1}{2} \times \frac{1}{16 \pi^{2}} c_{\infty} U_{0}\left(U_{0}^{\dagger} Y_{b}^{*} U_{0}^{*}\left(W_{0}\right)_{b a}\right)\left(U_{0}^{T} Y_{c} U_{0}\left(W_{0}\right)_{c a}\right) U_{0}^{\dagger}
\end{aligned}
$$




$$
=-\frac{1}{2} \times \frac{1}{16 \pi^{2}} c_{\infty} Y_{a}^{*} Y_{a}
$$

In the case of the divergence in $\Sigma_{L}^{(B)}$ - see equation (44C), we have to use the slightly different transformation

$$
U_{0}^{*} \hat{Y}_{a} \hat{m}_{0} \hat{Y}_{a} U_{0}^{\dagger}=Y_{a} m_{0}^{*} Y_{a}
$$

This explains that we have to be careful when a fermion mass term occurs because in general

$$
v_{a} Y_{a}^{*}=m_{0}^{*} \neq v_{a} Y_{a}=m_{0} .
$$

This complication only arises in

$$
\left(T_{\infty}^{(\chi)}\right)_{a}=\frac{1}{16 \pi^{2}} c_{\infty} \operatorname{Tr}\left[Y_{a} m_{0}{ }^{*} m_{0} m_{0}{ }^{*}+Y_{a}^{*} m_{0} m_{0}{ }^{*} m_{0}\right]
$$

and

$$
\left(\Pi_{\infty}^{(a)}\right)_{a b}=\frac{1}{16 \pi^{2}} c_{\infty} \operatorname{Tr}\left[Y_{a} m_{0}^{*} Y_{b} m_{0}^{*}+Y_{a}^{*} m_{0} Y_{b}^{*} m_{0}+2 Y_{a} Y_{b}^{*} m_{0} m_{0}^{*}+2 Y_{a}^{*} Y_{b} m_{0}{ }^{*} m_{0}\right]
$$

The divergences $\left(T_{\infty}^{(h)}\right)_{a},\left(\Pi_{\infty}^{(b)}\right)_{a b}$ and $\left(\Pi_{\infty}^{(c)}\right)_{a b}$ are obtained in a general basis by simply removing the hats from all quantities and the same is true for all counterterms.

\section{An example of a flavour symmetry}

Motivated by flavour models of the lepton sector [3], we will now consider a Lagrangian with a simple flavour symmetry and study how renormalization is affected in this case.

\subsection{Symmetry group and Lagrangian}

We assume the same number of Majorana and scalar fields, i.e. $n_{\chi}=n_{\varphi} \equiv n$. In addition, we require $n \geq 2$. Instead of the $\mathbb{Z}_{4}$ symmetry of equation (2), which acts at the same time on all fields, we will now postulate a $\mathbb{Z}_{4}$ symmetry for every index $a=1, \ldots, n$ :

$$
\left(\mathbb{Z}_{4}\right)_{a}: \quad \chi_{a L}^{(B)} \rightarrow i \chi_{a L}^{(B)}, \quad \varphi_{a}^{(B)} \rightarrow-\varphi_{a}^{(B)}, \quad \chi_{b L}^{(B)} \rightarrow \chi_{b L}^{(B)}, \quad \varphi_{b}^{(B)} \rightarrow \varphi_{b}^{(B)} \forall b \neq a .
$$

This has the consequence that scalar fields with the same index occur in pairs in the scalar potential. Note that now it is reasonable to use the same indices for both fermions and scalars. In addition, we assume that the Lagrangian is invariant under simultaneous permutations of fermion and scalar fields. Therefore, group-theoretically the symmetry group of the Lagrangian can be conceived as

$$
G_{n}=\left(\mathbb{Z}_{4}\right)^{n} \rtimes S_{n}
$$

With this flavour group, the bare Lagrangian has the form

$$
\mathcal{L}_{B}=\sum_{a=1}^{n}\left[i \bar{\chi}_{a L}^{(B)} \not \partial \chi_{a L}^{(B)}+\frac{1}{2} \partial_{\mu} \varphi_{a}^{(B)} \partial^{\mu} \varphi_{a}^{(B)}+\frac{1}{2} y^{(B)}\left(\chi_{a L}^{(B)} C^{-1} \chi_{a L}^{(B)} \varphi_{a}^{(B)}+\text { H.c. }\right)\right]-V_{B},
$$


where the bare scalar potential can be written as

$$
V_{B}=\frac{1}{2} \mu^{2} \sum_{a=1}^{n}\left(\varphi_{a}^{(B)}\right)^{2}+\frac{1}{4} \lambda\left(\sum_{a=1}^{n}\left(\varphi_{a}^{(B)}\right)^{2}\right)^{2}+\frac{1}{4} \lambda^{\prime} \sum_{a, b=1}^{n}\left(\varphi_{a}^{(B)}\right)^{2}\left(\varphi_{b}^{(B)}\right)^{2}\left(1-\delta_{a b}\right),
$$

where $\delta_{a b}$ is the Kronecker delta.

\subsection{Relation to the general model}

Due to the symmetry group $G_{n}$, we only have one Yukawa coupling constant and two quartic couplings. In order to use the general one-loop results, we have to establish the relation between the general model of section (2.1) and the present example. For simplicity we now drop the superscript $(B)$ and keep in mind that the following list applies not only to the renormalized coupling constants but also to the counterterms and the bare coupling constants:

$$
\begin{aligned}
\left(Y_{a}\right)_{b c} & =y \delta_{a b} \delta_{a c} \quad \forall a, \\
\left(\mu^{2}\right)_{a b} & =\mu^{2} \delta_{a b}, \\
\lambda_{a a a a} & =\lambda \quad \forall a \quad \text { and } \quad \lambda_{a a b b}=\frac{1}{3}\left(\lambda+\lambda^{\prime}\right) \quad \forall a \neq b .
\end{aligned}
$$

Note that now we just have one mass parameter $\mu^{2}$. Moreover, quartic couplings $\lambda_{a b b b}$ with $a \neq b$ and those with three or four different indices are zero. Without loss of generality we assume $y>0$. In addition, we have to consider equation (11), which now reads

$$
\delta_{a b}^{(\chi)}=\delta^{(\chi)} \delta_{a b}, \quad \delta_{a b}^{(\varphi)}=\delta^{(\varphi)} \delta_{a b},
$$

because due to the symmetry group $G_{n}$ only one field strength renormalization constant is allowed for each type of fields.

The results of section 4, found for the general Yukawa model, can directly be used for the present case by applying equation (73). In this way we obtain the counterterms

$$
\begin{aligned}
\delta y & =\frac{1}{16 \pi^{2}} c_{\infty} y^{3} \\
\delta \lambda^{(\chi)} & =-\frac{2}{16 \pi^{2}} c_{\infty} y^{4} \\
\delta \lambda^{(\varphi)} & =\frac{1}{16 \pi^{2}} c_{\infty}\left[9 \lambda^{2}+(n-1)\left(\lambda+\lambda^{\prime}\right)^{2}\right], \\
\left(\delta \lambda+\delta \lambda^{\prime}\right)^{(\chi)} & =0, \\
\left(\delta \lambda+\delta \lambda^{\prime}\right)^{(\varphi)} & =\frac{1}{16 \pi^{2}} c_{\infty}\left[6 \lambda\left(\lambda+\lambda^{\prime}\right)+(n+2)\left(\lambda+\lambda^{\prime}\right)^{2}\right], \\
\delta \mu^{2} & =\frac{\mu^{2}}{16 \pi^{2}} c_{\infty}\left[3 \lambda+(n-1)\left(\lambda+\lambda^{\prime}\right)\right],
\end{aligned}
$$

where the superscripts $(\chi)$ and $(\varphi)$ indicate fermions and scalars in the loop, respectively, in analogy to the notation in section 4.2. Field strength renormalization yields

$$
\delta^{(\chi)}=-\frac{1}{2} \times \frac{1}{16 \pi^{2}} c_{\infty} y^{2} \quad \text { and } \quad \delta^{(\varphi)}=-\frac{1}{16 \pi^{2}} c_{\infty} y^{2}
$$




\subsection{Spontaneous symmetry breaking}

In order to have SSB we assume $\mu^{2}<0$. For the vacuum expectation values we introduce the notation

$$
v^{2}=\sum_{a=1}^{n} v_{a}^{2}
$$

Obviously, for the scalar potential to be bounded from below we must have $\lambda>0$, but $\lambda^{\prime}$ can be positive or negative.

Case $\lambda^{\prime}>0$ : Here, the minimum of the scalar potential is achieved when only one VEV is nonzero. Without loss of generality we assume

$$
v_{1}=v, \quad v_{2}=\cdots=v_{n}=0 \quad \Rightarrow \quad v^{2}=-\frac{\mu^{2}}{\lambda} .
$$

The symmetry breaking can be formulated as

$$
G_{n} \stackrel{\text { SSB }}{\longrightarrow} G_{n-1}
$$

where $G_{n-1}$ is the residual symmetry group. This residual symmetry is reflected in the mass spectrum

$$
M_{01}^{2}=2 \lambda v^{2}, M_{02}^{2}=\cdots M_{0 n}^{2}=\lambda^{\prime} v^{2}, \quad m_{01}=y v, m_{02}=\cdots=m_{0 n}=0 .
$$

Since the mass matrices of both fermions and scalars are diagonal at tree level, it is straightforward to compute the one-loop corrections to equation (80). It easy to see that at one-loop order the VEV shifts fulfill $\Delta v_{2}=\cdots=\Delta v_{n}=0$, only $\Delta v_{1}$ will in general be nonzero. It is also obvious that the nonzero masses in equation (80) receive one-loop corrections. However, $m_{2}=\cdots=m_{n}$ is still valid because the unbroken symmetry group $G_{n-1}$ forbids such masses.

Case $\lambda^{\prime}<0$ : For negative $\lambda^{\prime}$, the condition

$$
\left|\lambda^{\prime}\right|<\frac{n}{n-1} \lambda
$$

is necessary for the scalar potential to be bounded from below. In this case the minimum is given by

$$
v_{1}^{2}=\cdots=v_{n}^{2}=\frac{v^{2}}{n} \quad \Rightarrow \quad v^{2}=\frac{-\mu^{2}}{\lambda+\frac{n-1}{n} \lambda^{\prime}} .
$$

In principle, the VEVs $v_{a}$ could have different signs. However, since arbitrary sign changes of the scalar fields are part of $G_{n}$, we can assume $v_{a}>0 \forall a$ without loss of generality. Therefore, we have the symmetry breaking

$$
G_{n} \stackrel{\mathrm{SSB}}{\longrightarrow} S_{n}
$$

where the permutation group is given by its "natural permutation representation" corresponding to $n \times n$ permutation matrices. This representation decays into the trivial 
one-dimensional and a $(n-1)$-dimensional irreducible representation. Defining $n$ vectors $w_{a}(a=1, \ldots, n)$ such that

$$
w_{1}=\frac{1}{\sqrt{n}}\left(\begin{array}{c}
1 \\
1 \\
\vdots \\
1
\end{array}\right) \quad \text { and } \quad w_{a} \cdot w_{b}=\delta_{a b} \forall a, b
$$

then $w_{1}$ is invariant under all permutation matrices and belongs, therefore, to the trivial irreducible representation, while the vectors $w_{2}, \ldots w_{n}$ span the space pertaining to the $(n-1)$-dimensional one. This is borne out by the tree-level masses. The scalars have the mass matrix

$$
M_{0}^{2}=A \mathbb{1}+B w_{1} w_{1}^{T} \quad \text { with } \quad A=-\frac{2 \lambda^{\prime} v^{2}}{n}, \quad B=2\left(\lambda+\lambda^{\prime}\right) v^{2} .
$$

Hence, the diagonalization matrix is given by

$$
W_{0}=\left(w_{1}, \ldots, w_{n}\right)
$$

and we find

$$
M_{01}^{2}=A+B, \quad M_{02}^{2}=\cdots=M_{0 n}^{2}=A .
$$

However, the fermion masses are all equal at tree level:

$$
m_{01}=\cdots=m_{0 n}=\frac{y v}{\sqrt{n}}
$$

At one-loop order, the scalar masses of equation (87) will receive radiative corrections, but - due to the unbroken symmetry group $S_{n}$-the relation $M_{2}^{2}=\cdots=M_{n}^{2}$ will still hold.

One might expect that the total degeneracy of the fermion masses, as expressed in equation (88), will be lifted because of radiative corrections such that $m_{1}$ is different from the rest. However, as we will demonstrate now, this is not the case.

First we discuss the contribution from the finite one-loop VEVs shifts to the fermion masses. Since the fermion mass matrix is diagonal, we have

$$
\hat{Y}_{a}=Y_{b}\left(W_{0}\right)_{b a}=y \operatorname{diag}\left(\left(W_{0}\right)_{1 a}, \ldots,\left(W_{0}\right)_{n a}\right) .
$$

In particular,

$$
\hat{Y}_{1}=\frac{y}{\sqrt{n}} \mathbb{1} \quad \text { and } \quad \operatorname{Tr} \hat{Y}_{a}=0 \quad \text { for } a=2, \ldots, n
$$

due to $w_{1}$ of equation (84). Therefore, it follows from equation (150) that

$$
T_{a}^{(\chi)}=0 \quad \text { for } a=2, \ldots, n .
$$

Moreover, from equations (22d $)$ and (86) we find

$$
\hat{v}_{1}=v, \quad \hat{v}_{2}=\cdots=\hat{v}_{n}=0 .
$$


With this the tadpole expression $T_{a}^{(h)}$ of equation (51) has the structure

$$
T_{a}^{(h)}=\hat{\lambda}_{a b r r} \hat{v}_{b} X_{r}=\hat{\lambda}_{a 1 r r} v X_{r}
$$

According to equation (73c), this expression can only be nonzero for $a=1$. Therefore,

$$
T_{a}^{(h)}=0 \quad \text { for } a=2, \ldots, n
$$

as well and $\Delta \hat{v}_{a} \hat{Y}_{a}=\Delta \hat{v}_{1} \hat{Y}_{1} \propto \mathbb{1}$. This proves that the finite VEV shifts cannot remove the total fermion mass degeneracy.

Next we consider $\Sigma^{1 \text {-loop }}$ of equation (44). We note that both $\hat{D}_{a}$ and $\hat{E}_{a}$ are proportional to the unit matrix because of equation (88). In addition, because of equation (87),

$$
\hat{D}_{2}=\ldots=\hat{D}_{n} \quad \text { and } \quad \hat{E}_{2}=\ldots=\hat{E}_{n} .
$$

Thus we can write $\hat{D}_{a}=f_{a} \mathbb{1}$ with $f_{2}=\cdots=f_{n}$, but $f_{1} \neq f_{2}$ in general. There are the analogous relations for the $\hat{E}_{a}$. Considering now the $b$-th entry of the (diagonal) finite parts of $\Sigma^{1 \text {-loop }}$ and taking into account that the Yukawa coupling matrices are given by equation (89), we have the generic sum

$$
\sum_{a=1}^{n}\left(W_{0}\right)_{b a} f_{a}\left(W_{0}\right)_{b a}=\left(W_{0}\right)_{b 1}\left(f_{1}-f_{2}\right)\left(W_{0}\right)_{b 1}+\sum_{a=1}^{n}\left(W_{0}\right)_{b a} f_{2}\left(W_{0}\right)_{b a}=\frac{1}{n}\left(f_{1}-f_{2}\right)+f_{2} .
$$

(Note that there is no summation over the index $b$ in this equation.) This result does not depend on $b$ and, therefore, $\Sigma^{1-\text { loop }}$ is proportional to the unit matrix. Consequently, the fermion mass degeneracy cannot be lifted by one-loop contributions, as stated above.

\subsection{Soft symmetry breaking}

It is possible to lift any mass degeneracies by explicit breaking of $G_{n}$. The model remains renormalizable, if we have soft breaking, for instance, by terms of dimension two. This is done by admitting in equation (73) a general mass matrix $\mu_{a b}^{2}$, whereas the Yukawa and quartic couplings are still restricted by $G_{n}$. This breaks the symmetry group $G_{n}$ down to

$$
G \stackrel{\operatorname{dim} 2}{\longrightarrow}\left(\mathbb{Z}_{4}\right)_{\operatorname{diag}}
$$

with

$$
\left(\mathbb{Z}_{4}\right)_{\text {diag }}: \quad \chi_{a L}^{(B)} \rightarrow i \chi_{a L}^{(B)}, \quad \varphi_{a}^{(B)} \rightarrow-\varphi_{a}^{(B)} \forall a,
$$

i.e. this $\mathbb{Z}_{4}$ acts simultaneously on all fields and agrees with equation (2). In this way, the scalar mass spectrum will be completely non-degenerate already at tree level, but also the fermion mass spectrum because a general matrix $\mu_{a b}^{2}$ will induce general VEVs $v_{a}$. It is easy to understand why this modified model remains renormalizable; allowing for a general matrix $\mu_{a b}^{2}$, we also allow for a general counterterm matrix $\delta \mu_{a b}^{2}$ and we can cancel the divergences related to the scalar mass terms as handled by equation (59).

It is natural that soft symmetry breaking is small. We can easily incorporate this by taking one large mass parameter $\mu^{2}$ and setting

$$
\mu_{a b}^{2}=\mu^{2} \delta_{a b}+\sigma_{a b}
$$


such that $\sum_{a=1}^{n} \sigma_{a a}=0$ and $\left|\sigma_{a b}\right| \ll \mu^{2} \forall a, b$. In this case the previously degenerate masses will now become slightly different and we can produce quasi-degenerate mass spectra.

\section{Dirac fermions}

So far, we have put the focus on Majorana fermions. We have done so because in the long run we are interested in studying radiative corrections in neutrino mass models which typically feature the seesaw mechanism and, therefore, neutrinos of Majorana nature. However, it is straightforward to switch from Majorana to Dirac fermions. How this is done will be explained in this section-see also [14, 19].

Lagrangian, diagonalization of Dirac mass matrices, and renormalization: In the Dirac setup, we can in general have $n_{\chi_{L}}$ chiral fields $\chi_{i L}^{(B)}$ and $n_{\chi_{R}}$ independent chiral fields $\chi_{i R}^{(B)}$, while the scalar sector remains the same as in the Majorana case. Then, the bare Lagrangian is given by

$$
\begin{aligned}
\mathcal{L}_{B}= & i \bar{\chi}_{i L}^{(B)} \gamma^{\mu} \partial_{\mu} \chi_{i L}^{(B)}+i \bar{\chi}_{i R}^{(B)} \gamma^{\mu} \partial_{\mu} \chi_{i R}^{(B)}+\frac{1}{2}\left(\partial_{\mu} \varphi_{a}^{(B)}\right)\left(\partial^{\mu} \varphi_{a}^{(B)}\right) \\
& -\left(\left(Y_{a}^{(B)}\right)_{i j} \bar{\chi}_{i R}^{(B)} \chi_{j L}^{(B)} \varphi_{a}^{(B)}+\text { H.c. }\right) \\
& -\frac{1}{2}\left(\mu_{B}^{2}\right)_{a b} \varphi_{a}^{(B)} \varphi_{b}^{(B)}-\frac{1}{4} \lambda_{a b c d}^{(B)} \varphi_{a}^{(B)} \varphi_{b}^{(B)} \varphi_{c}^{(B)} \varphi_{d}^{(B)}
\end{aligned}
$$

where the $Y_{a}^{(B)}$ now are $n_{\varphi}$ general complex $n_{\chi_{R}} \times n_{\chi_{L}}$ matrices. In principle, $n_{\chi_{L}}$ could be different from $n_{\chi_{R}}$, in which case one has $\left|n_{\chi_{L}}-n_{\chi_{R}}\right|$ massless Weyl fermions. However, for simplicity we assume $n_{\chi_{L}}=n_{\chi_{R}} \equiv n_{\chi}$ in the following. A possible modification of the transformation of the fermions in equation (2) is the $\mathbb{Z}_{2}$ symmetry

$$
\mathcal{S}^{\prime}: \quad \chi_{L}^{(B)} \rightarrow-\chi_{L}^{(B)}, \quad \chi_{R}^{(B)} \rightarrow \chi_{R}^{(B)}, \quad \varphi^{(B)} \rightarrow-\varphi^{(B)},
$$

in order to forbid fermion tree-level mass terms and linear and trilinear terms in the scalar potential.

The renormalization of the fermionic fields now becomes

$$
\chi_{L}^{(B)}=Z_{\chi_{L}}^{(1 / 2)} \chi_{L}, \quad \chi_{R}^{(B)}=Z_{\chi_{R}}^{(1 / 2)} \chi_{R},
$$

involving two independent general complex matrices $Z_{\chi_{L}}^{(1 / 2)}$ and $Z_{\chi_{R}}^{(1 / 2)}$. Inserting this into equation (100) yields a renormalized Lagrangian with Yukawa coupling matrices $Y_{a}$ and counterterms similar to the Majorana case. The main changes lie in the definition of the Yukawa counterterm

$$
\mathcal{M}^{\varepsilon / 2} \delta Y_{a}=\left(Z_{\chi_{R}}^{(1 / 2)}\right)^{\dagger} Y_{b}^{(B)} Z_{\chi_{L}}^{(1 / 2)}\left(Z_{\varphi}^{(1 / 2)}\right)_{b a}-\mathcal{M}^{\varepsilon / 2} Y_{a},
$$

and the need for the definition of two independent hermitian matrices

$$
\delta^{\left(\chi_{L}\right)}=\left(Z_{\chi_{L}}^{(1 / 2)}\right)^{\dagger} Z_{\chi_{L}}^{(1 / 2)}-\mathbb{1}, \quad \delta^{\left(\chi_{R}\right)}=\left(Z_{\chi_{R}}^{(1 / 2)}\right)^{\dagger} Z_{\chi_{R}}^{(1 / 2)}-\mathbb{1} .
$$


Via SSB we obtain the tree-level Dirac mass matrix

$$
m_{0}=\sum_{a=1}^{n_{\varphi}} v_{a} Y_{a}
$$

This mass matrix is bi-diagonalized with two unitary matrices $U_{L 0}$ and $U_{R 0}$ :

$$
U_{R 0}^{\dagger} m_{0} U_{L 0}=\hat{m}_{0} \equiv \operatorname{diag}\left(m_{01}, \ldots, m_{0 n_{\chi}}\right) \text {. }
$$

Due to the left and right diagonalization matrices, there are now left and right chiral mass eigenfields

$$
\hat{\chi}_{L}=U_{L 0}^{\dagger} \chi_{L}, \quad \hat{\chi}_{R}=U_{R 0}^{\dagger} \chi_{R} .
$$

Moreover, equations (22a) and (22b) are modified to

$$
\begin{aligned}
\delta^{\left(\chi_{L}\right)} & \rightarrow \hat{\delta}^{\left(\chi_{L}\right)}=U_{L 0}^{\dagger} \delta^{\left(\chi_{L}\right)} U_{L 0}, \quad \delta^{\left(\chi_{R}\right)} \rightarrow \hat{\delta}^{\left(\chi_{R}\right)}=U_{R 0}^{\dagger} \delta^{\left(\chi_{R}\right)} U_{R 0} \\
Y_{a} & \rightarrow \hat{Y}_{a}=\left(U_{R 0}^{\dagger} Y_{b} U_{L 0}\right)\left(W_{0}\right)_{b a}
\end{aligned}
$$

respectively.

In analogy to equation (24), we define Dirac mass eigenfields

$$
\hat{\chi}_{i}=\hat{\chi}_{i L}+\hat{\chi}_{i R}
$$

and the corresponding vector of eigenfields $\hat{\chi}$. In terms of mass eigenfields, the Yukawa interaction reads

$$
\mathcal{L}_{Y}=-\overline{\hat{\chi}}\left(\hat{Y}_{a} \gamma_{L}+\hat{Y}_{a}^{\dagger} \gamma_{R}\right) \hat{\chi}\left(\mathcal{M}^{\varepsilon / 2} \hat{h}_{a}+\hat{\bar{v}}_{a}\right)
$$

Formally, Dirac and Majorana Yukawa terms look the same [19]. Note that the only difference of this $\mathcal{L}_{Y}$ to that of equation (23) is the factor $1 / 2$ which we do not introduce in the Dirac case. It will become clear in the last paragraph of this section why we prefer this definition.

Fermion selfenergy: With the above definitions, the renormalization programme of section 3 goes through with only minor modifications. The renormalized fermion selfenergy for Dirac fermions is given by

$$
\begin{aligned}
\Sigma(p)= & \Sigma^{1-\text { loop }}(p)-\not p\left[\hat{\delta}^{\left(\chi_{L}\right)} \gamma_{L}+\hat{\delta}^{\left(\chi_{R}\right)} \gamma_{R}\right] \\
& +\hat{v}_{a}\left[\delta \hat{Y}_{a} \gamma_{L}+\left(\delta \hat{Y}_{a}\right)^{\dagger} \gamma_{R}\right]+\Delta \hat{v}_{a}\left[\hat{Y}_{a} \gamma_{L}+\hat{Y}_{a}^{\dagger} \gamma_{R}\right]
\end{aligned}
$$

Eventually, the one-loop Dirac masses read

$$
m_{i}=m_{0 i}+\frac{1}{2} m_{0 i}\left[\left(\Sigma_{L}^{(A)}\right)_{i i}\left(m_{0 i}^{2}\right)+\left(\Sigma_{R}^{(A)}\right)_{i i}\left(m_{0 i}^{2}\right)\right]+\operatorname{Re}\left(\Sigma_{L}^{(B)}\right)_{i i}\left(m_{0 i}^{2}\right) .
$$

Note that $\operatorname{Re}\left(\Sigma_{L}^{(B)}\right)_{i i}=\operatorname{Re}\left(\Sigma_{R}^{(B)}\right)_{i i}$ because of the symmetry relation (A1). 
Computation of amplitudes: There are two changes when we switch from Majorana to Dirac fermions [14]:

i. $\hat{Y}_{a}^{*} \rightarrow \hat{Y}_{a}^{\dagger}$ and

ii. a factor of two for every closed Dirac fermion loop compared to the corresponding Majorana fermion loop.

As discussed above, the first change simply comes from the fact that for Dirac neutrinos the Yukawa coupling matrices are not symmetric. The reason for the factor of two is the following. In the Majorana case we have defined the Yukawa Lagrangian with a factor $1 / 2$ - see equation (23). If a Majorana fermion line in a Feynman diagram is not closed, then all factors of $1 / 2$ are cancelled because, whenever a fermion line is connected to a vertex, there are two possible Wick contractions; however, in a closed loop one factor $1 / 2$ is left over because, when closing the loop, there is only one contraction. In the Dirac case, we have omitted the factor $1 / 2$ in the Yukawa Lagrangian (110) because, when we connect a Dirac fermion line to a vertex, there is exactly one Wick contraction. Therefore, when a closed fermion loop occurs, there is a factor of two for Dirac fermions relative to Majorana fermions. Finally, whenever we have made a simplification in a trace by exploiting $Y_{a}^{T}=Y_{a}$ in the Majorana case, as done in equations (47a) and (54), we have to revoke it in the Dirac case.

Consequently, in the Dirac case, $\Pi^{(a)}\left(p^{2}\right)$ is given by

$$
\begin{aligned}
\Pi_{a b}^{(a)}\left(p^{2}\right)= & \frac{2}{16 \pi^{2}}\left\{c _ { \infty } \operatorname { T r } \left[\hat{Y}_{a} \hat{m}_{0} \hat{Y}_{b} \hat{m}_{0}+\hat{Y}_{a}^{\dagger} \hat{m}_{0} \hat{Y}_{b}^{\dagger} \hat{m}_{0}+\hat{Y}_{a} \hat{Y}_{b}^{\dagger} \hat{m}_{0}^{2}+\hat{Y}_{a}^{\dagger} \hat{Y}_{b} \hat{m}_{0}^{2}\right.\right. \\
& \left.+\hat{Y}_{a} \hat{m}_{0}^{2} \hat{Y}_{b}^{\dagger}+\hat{Y}_{a}^{\dagger} \hat{m}_{0}^{2} \hat{Y}_{b}\right]-\frac{1}{2} c_{\infty} \operatorname{Tr}\left[\hat{Y}_{a} \hat{Y}_{b}^{\dagger}+\hat{Y}_{a}^{\dagger} \hat{Y}_{b}\right] p^{2} \\
& \left.+\frac{1}{2} \operatorname{Tr}\left[\left(\hat{Y}_{a} \hat{Y}_{b}^{\dagger}+\hat{Y}_{b}^{\dagger} \hat{Y}_{a}+\hat{Y}_{a}^{\dagger} \hat{Y}_{b}+\hat{Y}_{b} \hat{Y}_{a}^{\dagger}\right)\left(\hat{m}_{0}^{2}-\frac{1}{6} p^{2}\right)\right]-\cdots\right\}
\end{aligned}
$$

The dots refer to the integral in equation (47a) where merely $Y_{a}^{*}$ has to be substituted by $Y_{a}^{\dagger}$. From equation (113), $\left(\Pi_{\infty}^{(a)}\right)_{a b}$ can be read off. Equation (54) is modified to

$$
\delta \hat{\lambda}_{a b c d}^{(\chi)}=-\frac{1}{3} \times \frac{1}{16 \pi^{2}} c_{\infty} \operatorname{Tr}\left[\hat{Y}_{a} \hat{Y}_{b}^{\dagger} \hat{Y}_{c} \hat{Y}_{d}^{\dagger}+\cdots+\hat{Y}_{a}^{\dagger} \hat{Y}_{b} \hat{Y}_{c}^{\dagger} \hat{Y}_{d}+\cdots\right]
$$

where the dots indicate the five non-trivial permutations of the indices $b, c, d$. No complications arise in equations (50), (57), (67) and (68); for Dirac fermions one simply has to multiply the right-hand side by a factor of two and replace complex conjugation by hermitian conjugation.

\section{Conclusions}

In this paper we have presented a versatile and simple renormalization procedure which is adapted to models which have SSB and a multitude of scalars. This renormalization programme takes seriously the nature of masses as functions of the parameters of the underlying model; therefore, physical masses have an expansion in perturbation theory 
just like any other observable. We have exemplified our renormalization procedure by discussing a general Yukawa model with an arbitrary number of fermion fields of Majorana or Dirac nature and an arbitrary number of real scalar fields; moreover, this toy model has the feature that tree-level fermion masses are generated by SSB of a cyclic group. In particular, we have explicitly computed the fermionic and scalar selfenergies and studied radiative corrections at the one-loop level to tree-level masses.

The main idea discussed in this paper is to split renormalization into a step in which UV divergent parts are cancelled by $\overline{\mathrm{MS}}$ renormalization of the parameters of the unbroken theory and a subsequent step in which finite corrections are performed to make the scalar one-point functions vanish and to obtain one-loop pole masses. We have presented the details of the cancellation of UV divergences and elucidated the role of tadpole diagrams in our renormalization procedure and their contributions to the masses. We have also applied our findings to a showcase model furnished with a non-Abelian flavour symmetry group.

A typical example where the renormalization procedure put forward in this paper can be applied is the lepton sector of the multi-Higgs-doublet Standard Model with an arbitrary number of right-handed neutrino singlets and flavour symmetries; this comprises the seesaw mechanism as well as light sterile neutrinos. A derivation of general formulae which permit to compute radiative corrections to tree-level predictions of masses and mixing angles in this rather general class of flavour models is in preparation.

\section{Acknowledgments}

M.L. is supported by the Austrian Science Fund (FWF), Project No. P28085-N27 and in part by the FWF Doctoral Program No. W1252-N27 Particles and Interactions. The authors thank H. Eberl, G. Ecker, M. Mühlleitner and H. Neufeld for stimulating discussions. M.L. also thanks D. Lechner and C. Lepenik for further helpful discussions. 


\section{A Selfenergies and on-shell renormalization}

Since for fermions the general relations

$$
\left(\Sigma_{L}^{(A)}\right)^{\dagger}=\Sigma_{L}^{(A)}, \quad\left(\Sigma_{R}^{(A)}\right)^{\dagger}=\Sigma_{R}^{(A)}, \quad\left(\Sigma_{L}^{(B)}\right)^{\dagger}=\Sigma_{R}^{(B)}
$$

are valid, $\sqrt{7}$ we see that for the finiteness of $\Sigma_{L}^{(A)}$ and $\Sigma_{R}^{(A)}$ the counterterm with the hermitian $\delta^{(\chi)}$ suffices. In addition, we remark that in the case of Majorana fermions the further conditions [19, 20]

$$
\left(\Sigma_{L}^{(A)}\right)^{T}=\Sigma_{R}^{(A)}, \quad\left(\Sigma_{L}^{(B)}\right)^{T}=\Sigma_{L}^{(B)}, \quad\left(\Sigma_{R}^{(B)}\right)^{T}=\Sigma_{R}^{(B)}
$$

hold. This is a general condition, but can also be seen explicitly in our one-loop result.

In order to switch from the renormalized Majorana selfenergy $\Sigma(p)$ and the bosonic selfenergy $\Pi\left(p^{2}\right)$ to the on-shell selfenergies $\widetilde{\Sigma}(p)$ and $\widetilde{\Pi}\left(p^{2}\right)$, respectively, we must allow for finite field strength renormalization matrices. Denoting these by

$$
\stackrel{\circ}{Z}_{\chi}^{(1 / 2)}=\mathbb{1}+\frac{1}{2} \stackrel{\circ}{z}_{\chi} \quad \text { and } \quad \stackrel{\circ}{Z}_{h}^{(1 / 2)}=\mathbb{1}+\frac{1}{2} \stackrel{\circ}{z}_{h}
$$

we have at one-loop order

$$
\begin{aligned}
\widetilde{\Sigma}(p)= & \Sigma(p)-\frac{1}{2} \not p\left[\left(\left(\stackrel{\circ}{z}_{\chi}\right)^{\dagger}+\stackrel{\circ}{z}_{\chi}\right) \gamma_{L}+\left(\left(\stackrel{\circ}{z}_{\chi}\right)^{\dagger}+\stackrel{\circ}{z}_{\chi}\right)^{*} \gamma_{R}\right] \\
& +\frac{1}{2}\left[\left(\left(\stackrel{\circ}{z}_{\chi}\right)^{T} \hat{m}_{0}+\hat{m}_{0} \stackrel{\circ}{z}_{\chi}\right) \gamma_{L}+\left(\left(\stackrel{\circ}{z}_{\chi}\right)^{T} \hat{m}_{0}+\hat{m}_{0} \stackrel{\circ}{z}_{\chi}\right)^{*} \gamma_{R}\right], \\
\widetilde{\Pi}_{a b}\left(p^{2}\right)= & \Pi_{a b}\left(p^{2}\right)-\frac{1}{2}\left[\left(\stackrel{\circ}{z}_{h}\right)^{T}+\stackrel{\circ}{z}_{h}\right]_{a b} p^{2}+\frac{1}{2}\left[\left(\stackrel{\circ}{z}_{h}\right)^{T} \hat{M}_{0}^{2}+\hat{M}_{0}^{2} \stackrel{\circ}{z}_{h}\right]_{a b} .
\end{aligned}
$$

It is important to note that we have no freedom for mass renormalization because in our scheme the masses are computed in terms of the renormalized parameters of the model. Due to the Majorana nature of the fermions under consideration, the relation

$$
\stackrel{\circ}{z}_{\chi} \equiv\left(\stackrel{\circ}{z}_{L}\right)_{i j}=\left(\stackrel{\circ}{z}_{R}\right)_{i j}^{*}
$$

holds for left and right-chiral fields. In $\widetilde{\Sigma}(p)$ this fact has been taken into account. Using the second relation in equation (A1) and the first relation in equation (A2), the on-shell conditions lead for $i \neq j$ to [6, 16, 17, 21]

$$
\begin{aligned}
& \frac{1}{2}\left(\stackrel{\circ}{z}_{\chi}\right)_{i j}= \\
& \quad-\frac{1}{m_{0 i}^{2}-m_{0 j}^{2}}\left[m_{0 j}^{2}\left(\Sigma_{L}^{(A)}\right)_{i j}+m_{0 i} m_{0 j}\left(\Sigma_{L}^{(A)}\right)_{j i}+m_{0 j}\left(\Sigma_{L}^{(B)}\right)_{j i}^{*}+m_{0 i}\left(\Sigma_{L}^{(B)}\right)_{i j}\right]_{p^{2}=m_{0 j}^{2}} .
\end{aligned}
$$

\footnotetext{
${ }^{7}$ Strictly speaking these relations hold only for the dispersive part of the selfenergy.
} 
Furthermore, for $i=j$ we obtain

$$
\begin{aligned}
& \operatorname{Re}\left(\stackrel{\circ}{z}_{\chi}\right)_{i i}= \\
& \left.\left.\quad\left(\Sigma_{L}^{(A)}\right)_{i i}\left(m_{0 i}^{2}\right)+2 m_{0 i}^{2} \frac{\mathrm{d}}{\mathrm{d} p^{2}}\left(\Sigma_{L}^{(A)}\right)_{i i}\left(p^{2}\right)\right)\left.\right|_{p^{2}=m_{0 i}^{2}}+2 m_{0 i} \frac{\mathrm{d}}{\mathrm{d} p^{2}} \operatorname{Re}\left(\Sigma_{L}^{(B)}\right)_{i i}\left(p^{2}\right)\right)\left.\right|_{p^{2}=m_{0 i}^{2}}
\end{aligned}
$$

and

$$
m_{0 i} \operatorname{Im}\left(\stackrel{\circ}{z}_{\chi}\right)_{i i}=-\operatorname{Im}\left(\Sigma_{L}^{(B)}\right)_{i i}\left(m_{0 i}^{2}\right) .
$$

It is characteristic of Majorana fermions that there is no phase freedom in the determination of the field strength renormalization matrix, i.e. not only the real part but also the imaginary part of $\left(z_{\chi}\right)_{i i}$ is fixed.

Finally, in the scalar scalar case we are lead to

$$
a \neq b: \frac{1}{2}\left(\stackrel{\circ}{z}_{h}\right)_{a b}=-\frac{\Pi_{a b}\left(M_{0 b}^{2}\right)}{M_{0 a}^{2}-M_{0 b}^{2}}, \quad a=b:\left(\stackrel{\circ}{z}_{h}\right)_{a a}=\left.\frac{\mathrm{d} \Pi_{a a}\left(p^{2}\right)}{\mathrm{d} p^{2}}\right|_{p^{2}=M_{0 a}^{2}}
$$

for on-shell renormalization.

\section{B Finite tadpole contributions}

Throughout this appendix the discussion refers to the one-loop order. In the fermionic as well as the scalar selfenergy, tadpole diagrams contribute indirectly via the finite shift (36), even though in both cases the condition $t_{a}=0$ of equation (18) and the requirement that the scalar one-point function is zero - see equation (32) - procure the vanishing of the sum of tadpole diagrams and the term

$$
\Delta \hat{t}_{a}+\delta \hat{\mu}_{a b}^{2} \hat{v}_{b}+\delta \hat{\lambda}_{a b c d} \hat{v}_{b} \hat{v}_{c} \hat{v}_{d}
$$

Diagrammatically, this can be written as

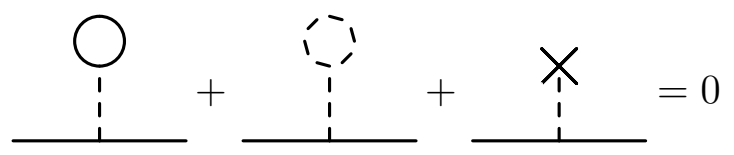

and

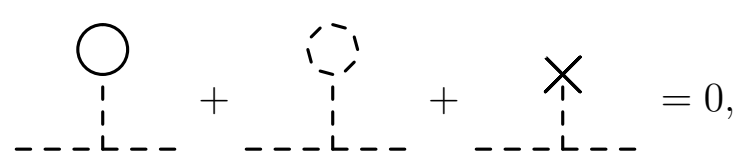

where the cross symbolizes the contribution of equation (B1). Still, the finite parts of the tadpole diagrams generate, via the finite VEV shifts $\Delta v_{a}$, the mass shifts

$$
\Delta \hat{m}_{0}=\hat{Y}_{a} \Delta \hat{v}_{a}
$$

for the fermions - see equation (28) - and

$$
\left(\Delta \hat{M}_{0}^{2}\right)_{a b}=6 \hat{\lambda}_{a b c d} \hat{v}_{c} \Delta \hat{v}_{d}
$$


for the real scalars - see equation (30). These add to the counterterms of the fermionic and scalar two-point functions. In terms of diagrams, this can be symbolized as

$$
\longrightarrow \quad=-i\left(\delta \hat{Y}_{a} \hat{v}_{a}+\Delta \hat{m}_{0}\right)
$$

for the fermions and

$$
---X--=-i\left(\delta \hat{\mu}_{a b}^{2}+3 \delta \hat{\lambda}_{a b c d} \hat{v}_{c} \hat{v}_{d}+\left(\Delta \hat{M}_{0}^{2}\right)_{a b}\right)
$$

for the scalars.

\section{Integrals}

$$
\begin{aligned}
\mathcal{M}^{\varepsilon} \int \frac{\mathrm{d}^{d} k}{(2 \pi)^{d}} \frac{1}{k^{2}-\Delta+i \epsilon} & =\frac{i}{16 \pi^{2}} \Delta\left(c_{\infty}+1-\ln \frac{\Delta}{\mathcal{M}^{2}}\right), \\
\mathcal{M}^{\varepsilon} \int \frac{\mathrm{d}^{d} k}{(2 \pi)^{d}} \frac{1}{\left(k^{2}-\Delta+i \epsilon\right)^{2}} & =\frac{i}{16 \pi^{2}}\left(c_{\infty}-\ln \frac{\Delta}{\mathcal{M}^{2}}\right), \\
\mathcal{M}^{\varepsilon} \int \frac{\mathrm{d}^{d} k}{(2 \pi)^{d}} \frac{k^{2}}{\left(k^{2}-\Delta+i \epsilon\right)^{2}} & =\frac{i}{16 \pi^{2}} \Delta\left(2 c_{\infty}+1-2 \ln \frac{\Delta}{\mathcal{M}^{2}}\right) .
\end{aligned}
$$




\section{References}

[1] C. Patrignani et al. (Particle Data Group), The Review of Particle Physics (2016), Chin. Phys. C 40 (2016) 100001.

[2] Y. Fukuda et al. [Super-Kamiokande Collaboration], Evidence for oscillation of atmospheric neutrinos, Phys. Rev. Lett. 81 (1998) 1562 hep-ex/9807003;

Q. R. Ahmad et al. [SNO Collaboration], Measurement of the rate of $\nu_{e}+d \rightarrow p+p+e^{-}$ interactions produced by ${ }^{8} B$ solar neutrinos at the Sudbury Neutrino Observatory, Phys. Rev. Lett. 87 (2001) 071301 [nucl-ex/0106015];

B. Aharmim et al. [SNO Collaboration], Combined analysis of all three phases of solar neutrino data from the Sudbury Neutrino Observatory, Phys. Rev. C 88 (2013) 025501 [arXiv:1109.0763 [nucl-ex]].

[3] S. F. King, Unified models of neutrinos, flavour and CP violation, Prog. Part. Nucl. Phys. 94 (2017) 217 arXiv:1701.04413 [hep-ph]];

F. Feruglio, Aspects of leptonic flavour mixing, Talk given at Neutrino 2016 (London, 4-9 July 2016) and Now 2016 (Otranto, 4-11 September 2016), arXiv:1611.09237 [hep$\mathrm{ph}$.

[4] S. Weinberg, Perturbative calculations of symmetry breaking, Phys. Rev. D 7 (1973) 2887.

[5] K. I. Aoki, Z. Hioki, M. Konuma, R. Kawabe and T. Muta, Electroweak theory. Framework of on-shell renormalization and study of higher order effects, Prog. Theor. Phys. Suppl. 73 (1982) 1.

[6] A. Denner and T. Sack, Renormalization of the quark mixing matrix, Nucl. Phys. B 347 (1990) 203.

[7] B. A. Kniehl and A. Pilaftsis, Mixing renormalization in Majorana neutrino theories, Nucl. Phys. B 474 (1996) 286 [hep-ph/9601390].

[8] J. Fleischer and F. Jegerlehner, Radiative corrections to Higgs decays in the extended Weinberg-Salam Model, Phys. Rev. D 23 (1981) 2001.

[9] A. Denner, L. Jenniches, J. N. Lang and C. Sturm, Gauge-independent $\overline{M S}$ renormalization in the 2HDM, JHEP 1609 (2016) 115 [arXiv:1607.07352 [hep-ph]].

[10] D. Pierce and A. Papadopoulos, Radiative corrections to the Higgs-boson decay rate $\Gamma(H \rightarrow Z Z)$ in the minimal supersymmetric model, Phys. Rev. D 47 (1993) 222 [hep-ph/9206257].

[11] M. Sperling, D. Stöckinger and A. Voigt, Renormalization of vacuum expectation values in spontaneously broken gauge theories, JHEP 1307 (2013) 132 arXiv:1305.1548 [hep-ph]]. 
[12] M. Krause, R. Lorenz, M. Mühlleitner, R. Santos and H. Ziesche, Gauge-independent renormalization of the 2-Higgs-doublet model, JHEP 1609 (2016) 143 arXiv:1605.04853 [hep-ph]].

[13] P. Minkowski, $\mu \rightarrow$ er at a rate of one out of $10^{9}$ muon decays?, Phys. Lett. 67B (1977) 421;

T. Yanagida, Horizontal gauge symmetry and masses of neutrinos, in Proceedings of the workshop on unified theory and baryon number in the universe (Tsukuba, Japan, 1979), O. Sawata and A. Sugamoto eds., KEK report 79-18, Tsukuba, 1979;

S.L. Glashow, The future of elementary particle physics, in Quarks and leptons, proceedings of the advanced study institute (Cargèse, Corsica, 1979), M. Lévy et al. eds., Plenum, New York, 1980;

M. Gell-Mann, P. Ramond and R. Slansky, Complex spinors and unified theories, in Supergravity, D.Z. Freedman and F. van Nieuwenhuizen eds., North Holland, Amsterdam, 1979;

R.N. Mohapatra and G. Senjanović, Neutrino mass and spontaneous parity violation, Phys. Rev. Lett. 44 (1980) 912.

[14] W. Grimus, P. O. Ludl and L. Nogués, Mass renormalization in a toy model with spontaneously broken symmetry, arXiv:1406.7795 [hep-ph].

[15] I. Schur, Ein Satz Ueber Quadratische Formen Mit Komplexen Koeffizienten, Am. J. Math. 67 (1945) 472.

[16] S. Kiyoura, M. M. Nojiri, D. M. Pierce and Y. Yamada, Radiative corrections to a supersymmetric relation: A new approach, Phys. Rev. D 58 (1998) 075002 hep-ph/9803210.

[17] W. Grimus and M. Löschner, Revisiting on-shell renormalization conditions in theories with flavor mixing, Int. J. Mod. Phys. A 31 (2016) 1630038; Erratum: ibid. A 32 (2017) 1792001 [arXiv:1606.06191 [hep-ph]].

[18] L. Altenkamp, S. Dittmaier and H. Rzehak, Renormalization schemes for the two-Higgs-doublet model and applications to $h \rightarrow W W / Z Z \rightarrow 4$ fermions, arXiv:1704.02645 [hep-ph].

[19] A. Denner, H. Eck, O. Hahn and J. Küblbeck, Compact Feynman rules for Majorana fermions, Phys. Lett. B 291 (1992) 278;

A. Denner, H. Eck, O. Hahn and J. Küblbeck, Feynman rules for fermion number violating interactions, Nucl. Phys. B 387 (1992) 467.

[20] W. Grimus and L. Lavoura, One-loop corrections to the seesaw mechanism in the multi-Higgs-doublet Standard Model, Phys. Lett. B 546 (2002) 86 [hep-ph/0207229].

[21] W. Grimus and L. Lavoura, Soft lepton-flavor violation in a multi-Higgs-doublet seesaw model, Phys. Rev. D 66 (2002) 014016 [hep-ph/0204070]. 